\title{
Odd-parity superconductivity by competing spin-orbit coupling and orbital effect in artificial heterostructures
}

\section{AUTHOR(S):}

Watanabe, Tatsuya; Yoshida, Tomohiro; Yanase, Youichi

\section{CITATION:}

Watanabe, Tatsuya ... [et al]. Odd-parity superconductivity by competing spin-orbit coupling and orbital effect in artificial heterostructures. Physical Review B 2015, 92(17): 174502.

ISSUE DATE:

2015-11-02

URL:

http://hdl.handle.net/2433/207616

RIGHT:

(C2015 American Physical Society 
PHYSICAL REVIEW B 92, 174502 (2015)

\title{
Odd-parity superconductivity by competing spin-orbit coupling and orbital effect in artificial heterostructures
}

\author{
Tatsuya Watanabe, ${ }^{1}$ Tomohiro Yoshida, ${ }^{2}$ and Youichi Yanase ${ }^{3, *}$ \\ ${ }^{1}$ Graduate School of Science and Technology, Niigata University, Niigata 950-2181, Japan \\ ${ }^{2}$ Department of Physics, Gakushuin University, Tokyo 171-8588, Japan \\ ${ }^{3}$ Department of Physics, Graduate School of Science, Kyoto University, Kyoto 606-8502, Japan
}

(Received 22 July 2015; published 2 November 2015)

\begin{abstract}
We show that odd-parity superconductivity occurs in multilayer Rashba systems without requiring spintriplet Cooper pairs. A pairing interaction in the spin-singlet channel stabilizes the odd-parity pair-densitywave (PDW) state in the magnetic field parallel to the two-dimensional conducting plane. It is shown that the layer-dependent Rashba spin-orbit coupling and the orbital effect play essential roles for the PDW state in binary and tricolor heterostructures. We demonstrate that the odd-parity PDW state is a symmetry-protected topological superconducting state characterized by the one-dimensional winding number in the symmetry class $B D I$. The superconductivity in the artificial heavy-fermion superlattice $\mathrm{CeCoIn}_{5} / \mathrm{YbCoIn}_{5}$ and bilayer interface $\mathrm{SrTiO}_{3} / \mathrm{LaAlO}_{3}$ is discussed.
\end{abstract}

DOI: 10.1103/PhysRevB.92.174502

PACS number(s): 74.20.Rp, 74.70.Tx, 74.78.Fk

\section{INTRODUCTION}

Parity is an essential quantum number of quantum phases unless inversion symmetry is broken. Classification of superconducting states is based on the parity of the order parameter [1]. According to the conventional understanding [1], evenparity superconductivity is realized by the condensation of spin-singlet Cooper pairs, while odd-parity superconductivity is induced by spin-triplet Cooper pairs because of the anticommutation relation of fermions. Even-parity superconductivity has been observed in a variety of materials, e.g., archetypal strongly correlated electron systems such as high- $T_{\mathrm{c}}$ cuprate superconductors (SCs) [2,3] as well as conventional SCs stabilized by electron-phonon coupling. On the other hand, only a few materials are considered as possible hosts of odd-parity superconductivity. This is probably because the conditions for spin-triplet pairing are unfavorable in most materials. Since electron-phonon coupling mostly stabilizes spin-singlet $s$-wave superconductivity, strong electron correlation is required for the glues of spin-triplet Cooper pairs. However, $d$-wave superconductivity is stable in most strongly correlated electron systems [4].

Odd-parity superconductivity has been attracting attention because of its multicomponent order parameters that give rise to multiple superconducting/superfluid phases and intriguing phenomena related to spontaneous symmetry breaking [1,5-9]. Furthermore, a great deal of attention has recently been paid to odd-parity SCs because they are candidates for topological superconductivity [10-15]. However, only $\mathrm{Sr}_{2} \mathrm{RuO}_{4}[8,9]$ and some uranium-based heavy-fermion compounds such as $\mathrm{UPt}_{3}[6,7], \mathrm{UGe}_{2}$ [16], URhGe, and UCoGe $[17,18]$ show strong evidence for spin-triplet pairing.

Recent theoretical studies have presented another way to stabilize the odd-parity superconducting state. It has been shown that odd-parity superconductivity may occur through spin-singlet Cooper pairs in crystals lacking local inversion symmetry. Such locally noncentrosymmetric crystals have a

*yanase@scphys.kyoto-u.ac.jp sublattice degree of freedom in electronic structures, allowing the odd-parity spin-singlet superconducting state [19,20]. Although this state is not allowed in the absence of spin-orbit coupling according to the BCS theory, such an exotic superconducting state may be stabilized by the sublattice-dependent spin-orbit coupling arising from the relativistic effect [20]. It has been shown that a long-range Coulomb interaction stabilizes odd-parity superconductivity in combination with spinorbit coupling $[21,22]$. On the other hand, two of the authors have shown that the odd-parity spin-singlet superconducting state is stabilized by spin-orbit coupling and the paramagnetic effect without relying on the particular electron correlation effect [23]. Therefore, conventional electron-phonon coupling or antiferromagnetic spin fluctuation leading to spin-singlet Cooper pairing may induce odd-parity superconductivity when both spin-orbit coupling and the paramagnetic effect play important roles.

In a previous study, we focused on two-dimensional (2D) multilayer SCs in which global inversion symmetry is preserved but some of the layers lack local inversion symmetry. Then, by applying a magnetic field along the $c$ axis, the order parameter of spin-singlet superconductivity changes sign across the center layer, as shown in Fig. 1(a) [23]. The order parameter spatially modulated in the atomic length scale ensures the odd parity of superconductivity. Such a superconducting state is called the pair-density-wave (PDW) state. Interestingly, the PDW state is classified into topological crystalline superconductivity protected by mirror reflection symmetry when the number of superconducting layers is odd [24]. A nontrivial topological invariant in the symmetry class $D$, the mirror Chern number ensures the appearance of the Majorana edge mode at the edge. A promising candidate for realizing such a topological superconducting state is the recently grown artificial superlattice $\mathrm{CeCoIn}_{5} / \mathrm{YbCoIn}_{5}$ composed of the quasi-2D heavy-fermion $\mathrm{SC} \mathrm{CeCoIn}_{5}$ and the conventional metal $\mathrm{YbCoIn}_{5}$ [25]. The superconductivity occurs in $\mathrm{CeCoIn}_{5}$ multilayers, and $\mathrm{YbCoIn}_{5}$ plays the role of spacer layers. Thus, the superlattice is regarded as a $2 \mathrm{D}$ multilayer $\mathrm{SC}$ when the number of $\mathrm{YbCoIn}_{5}$ layers is large. The strong spin-orbit coupling and the large paramagnetic 
(a) Binary superlattice

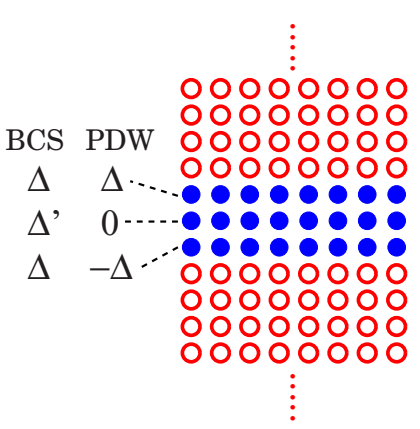

(b) Tricolor superlattice

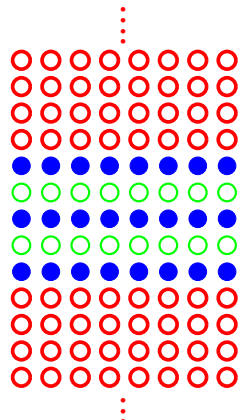

FIG. 1. (Color online) Illustration of superlattice structures studied in this paper. We show examples of the trilayer system $(M=3)$. (a) Binary superlattice that has been realized in $\mathrm{CeCoIn}_{5} / \mathrm{YbCoIn}_{5}$. Closed (blue) and open (red) circles show the superconducting layers and spacer layers, respectively. The layer-dependent order parameters in the BCS and PDW states are described on the left. (c) Tricolor superlattice. Thick (red) and thin (green) open circles show the two kinds of spacer layers.

effect [26,27] in $\mathrm{CeCoIn}_{5}$ are favorable for the topological odd-parity superconductivity discussed above. Indeed, the effects of layer-dependent Rashba spin-orbit coupling (RSOC) on the superconducting state of $\mathrm{CeCoIn}_{5} / \mathrm{YbCoIn}_{5}$ have been observed [28,29].

On the other hand, assuming a large Maki parameter $\alpha_{\mathrm{M}}=\sqrt{2} H_{\mathrm{c} 2}^{\mathrm{orb}} / H_{\mathrm{c} 2}^{\mathrm{P}}$, Ref. [23] neglected the orbital effect that competes with the paramagnetic effect. $H_{\mathrm{c} 2}^{\text {orb }}$ and $H_{\mathrm{c} 2}^{\mathrm{P}}$ are fictitious upper critical fields determined by the orbital effect (orbital limit) and by the paramagnetic effect (paramagnetic limit), respectively. Since the Maki parameter of bulk CeCoIn is moderate $\left(\alpha_{\mathrm{M}} \sim 3\right)$ for the magnetic field along the $c$ axis [30], the orbital effect may suppress the PDW state. A simple way to reduce the orbital effect is to apply a magnetic field along the $2 \mathrm{D}$ conducting plane. However, when the orbital effect is completely neglected, the in-plane magnetic field induces the complex stripe (CS) state [31], and the PDW state is not stabilized. In this paper, we show that the odd-parity PDW state is stabilized by switching on a weak orbital effect.

Furthermore, we find that the PDW state is a topological crystalline SC protected by magnetic mirror symmetry. Because mirror reflection symmetry with respect to the $a b$ plane is broken by the in-plane magnetic field, the mirror Chern number is no longer a topological invariant. However, magnetic mirror symmetry is preserved when we apply the magnetic field along the $a$ axis or the $b$ axis. Using this symmetry, we define the topological invariant, i.e., the one-dimensional winding number in the symmetry class $B D I$. We will show a nontrivial winding number and the resulting Majorana edge state.

The organization of this paper is as follows. In Sec. II we introduce the model for multilayer SCs possessing the layer-dependent RSOC. Various superconducting states obtained by solving the linearized mean-field equation are illustrated. We study the binary superlattices in Sec. III and discuss the superconducting state in the artificial superlattice $\mathrm{CeCoIn}_{5} / \mathrm{YbCoIn}_{5}$. The tricolor superlattice in Fig. 1(b) is also investigated in Sec. IV. In Secs. III and IV, it is shown that the

odd-parity PDW state is stabilized through the RSOC and the orbital effect. In Sec. V, the topologically nontrivial properties of the PDW state are clarified, and the Majorana edge state is demonstrated. A brief summary and discussions are provided in Sec. VI.

\section{MODEL AND FORMULATION}

\section{A. Model}

First, we introduce the model for 2D multilayer SCs. By simply neglecting the spacer layers, the binary and tricolor superlattices in Fig. 1 are described by the multilayer model. By taking into account the layer-dependent RSOC, the orbital effect, and Zeeman coupling, the Hamiltonian is described as

$$
\begin{aligned}
\mathcal{H}= & \sum_{\boldsymbol{k}, s, m} \xi\left(\boldsymbol{k}+\boldsymbol{p}_{m}\right) c_{\boldsymbol{k s m}}^{\dagger} c_{\boldsymbol{k} s m}+t_{\perp} \sum_{\boldsymbol{k}, s,\left\langle m, m^{\prime}\right\rangle} c_{\boldsymbol{k s m}}^{\dagger} c_{\boldsymbol{k s m ^ { \prime }}} \\
& +\sum_{\boldsymbol{k}, \boldsymbol{k}^{\prime}, \boldsymbol{q}, m} V\left(\boldsymbol{k}, \boldsymbol{k}^{\prime}\right) c_{\boldsymbol{k}_{+} \uparrow m}^{\dagger} c_{-\boldsymbol{k}_{-\downarrow m}}^{\dagger} c_{-\boldsymbol{k}_{-\downarrow}^{\prime} \downarrow} c_{\boldsymbol{k}_{+}^{\prime} \uparrow m} \\
& -\sum_{\boldsymbol{k}, s, s^{\prime}, m} \mu_{\mathrm{B}} \boldsymbol{H} \cdot \boldsymbol{\sigma}_{s s^{\prime}} c_{\boldsymbol{k} s m}^{\dagger} c_{\boldsymbol{k} s^{\prime} m} \\
& +\sum_{\boldsymbol{k}, s, s^{\prime}, m} \alpha_{m} \boldsymbol{g}\left(\boldsymbol{k}+\boldsymbol{p}_{m}\right) \cdot \boldsymbol{\sigma}_{s s^{\prime}} c_{\boldsymbol{k} s m}^{\dagger} c_{\boldsymbol{k} s^{\prime} m},
\end{aligned}
$$

where $\boldsymbol{k}, s$, and $m(=1, \ldots, M)$ are indexes of momentum, spin, and layer, respectively. The number of superconducting layers is $M$.

The first term is the energy dispersion in the single-layer limit. We adopt the nearest-neighbor hopping term in the square lattice for simplicity,

$$
\xi(\boldsymbol{k})=-2 t\left(\cos k_{x} a+\cos k_{y} a\right)-\mu,
$$

where $a$ is the lattice constant. The orbital effect induced by the applied magnetic field is taken into account through the Peierls phase. When we consider the magnetic field along the [100] axis, $\boldsymbol{H}=H \hat{x}$, we can choose the vector potential $\boldsymbol{A}=(0,-H z, 0)$. Then, the orbital effect leads to the layer-dependent shift of momentum, $\boldsymbol{k} \rightarrow \boldsymbol{k}+\frac{e}{\hbar} \boldsymbol{A}$. Thus, we obtain $\boldsymbol{p}_{m}=\frac{e}{\hbar} H d[m-(M+1) / 2] \hat{y}$, where $d$ is the lattice spacing between the nearest-neighbor superconducting layers. For binary superlattices, $d=c$, with $c$ being the lattice constant along the $c$ axis. Later we adopt the lattice constant of $\mathrm{CeCoIn}_{5}, a=4.6 \AA$, and $c=7.5 \AA$ since we focus on the artificial superlattice $\mathrm{CeCoIn}{ }_{5} / \mathrm{YbCoIn}_{5}$ [25]. The second term of Eq. (1) is the interlayer hopping term. Since we consider the heterostructures composed of quasi-2D SCs, the interlayer hopping $t_{\perp}$ is assumed to be much smaller than the in-plane hopping, $t_{\perp} \ll t$.

The third term describes the pairing interaction. We assume $s$-wave superconductivity for simplicity, and thus

$$
V\left(\boldsymbol{k}, \boldsymbol{k}^{\prime}\right)=-V_{\mathrm{s}} .
$$

We have confirmed that qualitatively the same results are obtained for $d$-wave superconductivity. As we will show layer, a spatially nonuniform superconducting state may be stabilized. Thus, we take into account the finite center-ofmass momentum of Cooper pairs $\boldsymbol{q}$, and $\boldsymbol{k}_{ \pm}=\boldsymbol{k} \pm \boldsymbol{q} / 2$. The 
fourth term is the Zeeman coupling term giving rise to the paramagnetic effect on the superconducting state.

We show that exotic superconducting states are stabilized by the layer-dependent RSOC represented in the last term of Eq. (1), which arises from the local violation of inversion symmetry [20]. Ensured by the global inversion symmetry in the crystal structure, the RSOC is odd with respect to the mirror reflection on the center layer, and thus $\alpha_{M+1-m}=-\alpha_{m}$. For example, the layer-dependent coupling constant is $\left(\alpha_{1}, \alpha_{2}\right)=$ $(\alpha,-\alpha)$ for bilayers and $\left(\alpha_{1}, \alpha_{2}, \alpha_{3}\right)=(\alpha, 0,-\alpha)$ for trilayers. We assume a $g$-vector characterizing the RSOC [32], $\boldsymbol{g}(\boldsymbol{k})=\left(-\sin k_{y} a, \sin k_{x} a, 0\right)$, so as to satisfy the periodicity in momentum space.

For bilayers, a similar model has been investigated by noticing the twin boundary of noncentrosymmetric SCs [33-36]. Then, the intriguing superconducting phase with broken time-reversal symmetry was investigated by assuming comparable pairing interactions for the spin-singlet Cooper pairs and spin-triplet ones [33-35]. We avoid such fine-tuning of pairing interactions here and consider the dominantly spinsinglet pairing state, which is realized in most SCs. Aoyama et al. studied the magnetoelectric effect on the upper and lower critical magnetic fields, [36] but their Ginzburg-Landau model does not appropriately take into account the paramagnetic effect in the high magnetic field region, and therefore the PDW state that is the focus of this paper is not obtained.

We assume small interlayer hopping $t_{\perp} / t=0.1$ and a moderate RSOC $\alpha / t=0.3$ unless explicitly mentioned otherwise. As shown by previous studies [20,23,31], exotic superconducting states may be stabilized when $|\alpha| / t_{\perp} \gtrsim 1$. Thus, we assume $|\alpha| / t_{\perp} \gtrsim 1$ throughout this paper. This condition may be satisfied in heterostructures of quasi-2D compounds. The chemical potential is $\mu / t=2$ in Secs. III and IV while it is $\mu / t=-2$ in Sec. V. We choose the pairing interaction $V_{\mathrm{s}} / t=1.3$ or 1.5 . We confirmed that the following results are almost independent of the choice of $V_{\mathrm{s}} / t$.

\section{B. Linearized mean-field theory}

We study the superconducting state by means of the linearized mean-field theory. Although we have to fully solve the Bogoliubov-de Gennes (BdG) equation in order to obtain the superconducting phase diagram, we can clarify the superconducting state near the transition temperature by linearizing the $\mathrm{BdG}$ equation while avoiding the numerical limitations of the full $\mathrm{BdG}$ equation. The linearized $\mathrm{BdG}$ equation is formulated by calculating the superconducting susceptibility,

$$
\chi_{m m^{\prime}}^{\mathrm{sc}}(q)=\int_{0}^{\beta} d \tau e^{i \Omega_{n} \tau}\left\langle B_{q m}(\tau) B_{q m^{\prime}}^{\dagger}(0)\right\rangle,
$$

where $q=\left(\boldsymbol{q}, i \Omega_{n}\right)$, and $\Omega_{n}=2 \pi n k_{\mathrm{B}} T$ is the boson Matsubara frequency. The annihilation operator of Cooper pairs is introduced as

$$
B_{q m}=\sum_{k} c_{k+q \uparrow m} c_{-k \downarrow m}
$$

and $B_{\boldsymbol{q} m}(\tau)=e^{-\mathcal{H} \tau} B_{\boldsymbol{q} m} e^{\mathcal{H} \tau}$.
The superconducting susceptibility is obtained by using the T-matrix approximation,

$$
\hat{\chi}^{\mathrm{sc}}(q)=\frac{\hat{\chi}^{0}(q)}{\hat{1}-V_{\mathrm{s}} \hat{\chi}^{0}(q)},
$$

where $\hat{\chi}^{\mathrm{sc}}=\left(\chi_{m m^{\prime}}^{\mathrm{sc}}\right)$ is the $M \times M$ matrix. The irreducible susceptibility is calculated by

$$
\begin{aligned}
\chi_{m m^{\prime}}^{0}(q)= & \frac{1}{\beta} \sum_{\boldsymbol{k}, l}\left[G_{m m^{\prime}}^{\uparrow \uparrow}\left(\boldsymbol{k}+\boldsymbol{q}, i \omega_{l}\right) G_{m m^{\prime}}^{\downarrow \downarrow}\left(-\boldsymbol{k}, i \Omega_{n}-i \omega_{l}\right)\right. \\
& \left.-G_{m m^{\prime}}^{\uparrow \downarrow}\left(\boldsymbol{k}+\boldsymbol{q}, i \omega_{l}\right) G_{m m^{\prime}}^{\downarrow \uparrow}\left(-\boldsymbol{k}, i \Omega_{n}-i \omega_{l}\right)\right], \quad
\end{aligned}
$$

where $G_{m m^{\prime}}^{s s^{\prime}}\left(\boldsymbol{k}, i \omega_{l}\right)$ is the noninteracting Green function and $\omega_{l}=(2 l+1) \pi k_{\mathrm{B}} T$ is the fermion Matsubara frequency.

The superconducting transition occurs at the temperature where $\hat{\chi}^{\mathrm{sc}}(q)$ diverges. Thus, the criterion of the superconducting instability is obtained as the largest eigenvalue of $V_{\mathrm{s}} \hat{\chi}^{0}(q)$ is unity. We obtain the layer-dependent order parameter $\Delta_{m}(\mathbf{r})=\Delta_{m} e^{i \mathbf{q} \cdot \mathbf{r}}$ from the eigenvector, $\left(\Delta_{1}, \Delta_{2}, \ldots, \Delta_{M}\right)^{\mathrm{T}}$. Because the global inversion symmetry is preserved in our model, the eigenvalues are equivalent between the momentum $\pm \mathbf{q}$. Hence, the single- $q$ state or the double- $q$ state may be stabilized when the center-of-mass momentum of Cooper pairs is finite. It is expected that the double- $q$ state is stable in our model because the order parameter almost disappears in one of the outermost layers in the single- $q$ state with a small condensation energy. For instance, $\Delta_{M} \ll \Delta_{1}$ for momentum $\boldsymbol{q}$ while $\Delta_{1} \ll \Delta_{M}$ for the opposite momentum $\boldsymbol{- q}$. In the double- $q$ state, the order parameter is described as $\Delta_{m}(\mathbf{r})=\Delta_{m}^{(+)} e^{i \mathbf{q} \cdot \mathbf{r}}+\Delta_{m}^{(-)} e^{-i \mathbf{q} \cdot \mathbf{r}}$, where $\Delta_{m}^{( \pm)}$is the eigenvector of $V_{\mathrm{s}} \hat{\chi}^{0}(q)$ for the momentum $\pm \mathbf{q}$, respectively. We confirmed that the bosonic Matsubara frequency is always zero, $\Omega_{n}=0$.

\section{Superconducting states}

In this subsection, we classify the solution of the linearized $\mathrm{BdG}$ equation. As we will show later, various superconducting states are stabilized in our model. They are illustrated for the bilayer system in Fig. 2. We discuss the bilayer system for simplicity, since the extension to more-than-two-layer systems is straightforward.

The uniform superconducting state $\left[\Delta_{m}(\mathbf{r})=\Delta\right]$ is stable at zero magnetic field as expected from conventional BCS theory. Thus, we call the uniform state the "BCS state" [Fig. 2(a)]. On

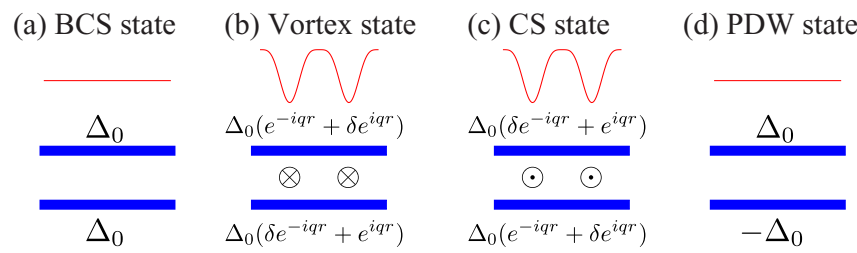

FIG. 2. (Color online) Illustration of superconducting states in the bilayer system. (a) BCS state, (b) vortex state, (c) CS state, and (d) PDW state. Thick bars show the superconducting layers. Layerdependent order parameters are described in the figures. The spatial dependence of the superconducting gap is illustrated by thin lines on top of the figures. The vortex in (b) and the antivortex in (c) are shown by arrows. 
the other hand, a variety of spatially nonuniform states may be stabilized in the magnetic field. First, the orbital effect induces the vortex state illustrated in Fig. 2(b). When the quantum vortices penetrate inside multilayers, the order parameter is described as

$$
\begin{aligned}
& \Delta_{1}(\mathbf{r})=\Delta_{0}\left(e^{-i \mathbf{q} \cdot \mathbf{r}}+\delta e^{i \mathbf{q} \cdot \mathbf{r}}\right), \\
& \Delta_{2}(\mathbf{r})=\Delta_{0}\left(\delta e^{-i \mathbf{q} \cdot \mathbf{r}}+e^{i \mathbf{q} \cdot \mathbf{r}}\right),
\end{aligned}
$$

where $|\delta|<1$, and $\mathbf{q}=q(\hat{z} \times \hat{H})$ with $\hat{H}=\boldsymbol{H} /|\boldsymbol{H}|$ and $q>$ 0 . Second, the layer-dependent RSOC stabilizes the CS state through the paramagnetic effect [31]. The CS state is also described by Eqs. (8) and (9), however the sign of the centerof-mass momentum $q$ depends on the band structure and the sign of RSOC. For our choice of parameters, the RSOC favors $q<0$ when $\alpha>0$ while $q>0$ when $\alpha<0$. Thus, the CS state is regarded as an antivortex state [Fig. 2(c)] and is distinguished from the vortex state when $\alpha>0$. Then the spin-orbit coupling competes with the orbital effect and gives rise to an intriguing superconducting phase diagram, as we show later. We focus on this case in the following, although the other case, $\alpha<0$, is briefly discussed.

Finally, Fig. 2(d) illustrates the PDW state, where $\left[\Delta_{1}(\mathbf{r}), \Delta_{2}(\mathbf{r})\right]=(\Delta,-\Delta)$ [23]. The order parameter is uniform in the $2 \mathrm{D}$ conducting plane, but it changes sign between layers. As we mentioned before, the PDW state is an oddparity superconducting state, although the superconductivity is induced by the spin-singlet $s$-wave Cooper pairs. Although we have shown that the PDW state is stabilized in the $c$-axis magnetic field near the Pauli limit [23], in this paper we show that the PDW state is also stabilized in the in-plane magnetic field when the RSOC competes with the orbital effect.

The BCS, PDW, vortex, and CS states are distinguished from each other in more-than-two-layer systems too. In Table I, we summarize the order parameter of these states in trilayers as well as in bilayers. The order parameter of the so-called Fulde-Ferrell-Larkin-Ovchinnikov (FFLO) state [37,38] is also shown for comparison.

TABLE I. Layer-dependent order parameter of the BCS, PDW, FFLO, vortex, and CS states in bilayers and trilayers.

\begin{tabular}{lll}
\hline \hline & \multicolumn{1}{c}{ Bilayer } & \multicolumn{1}{c}{ Trilayer } \\
\hline BCS & $\Delta_{1}(\mathbf{r})=\Delta$ & $\Delta_{1}(\mathbf{r})=\Delta$ \\
& $\Delta_{2}(\mathbf{r})=\Delta$ & $\Delta_{2}(\mathbf{r})=\Delta^{\prime}$ \\
& & $\Delta_{3}(\mathbf{r})=\Delta$ \\
PDW & $\Delta_{1}(\mathbf{r})=\Delta$ & $\Delta_{1}(\mathbf{r})=\Delta$ \\
& $\Delta_{2}(\mathbf{r})=-\Delta$ & $\Delta_{2}(\mathbf{r})=0$ \\
& & $\Delta_{3}(\mathbf{r})=-\Delta$ \\
FFLO & $\Delta_{1}(\mathbf{r})=\Delta \cos (\mathbf{q} \cdot \mathbf{r})$ & $\Delta_{1}(\mathbf{r})=\Delta \cos (\mathbf{q} \cdot \mathbf{r})$ \\
& $\Delta_{2}(\mathbf{r})=\Delta \cos (\mathbf{q} \cdot \mathbf{r})$ & $\Delta_{2}(\mathbf{r})=\Delta^{\prime} \cos (\mathbf{q} \cdot \mathbf{r})$ \\
& & $\Delta_{3}(\mathbf{r})=\Delta \cos (\mathbf{q} \cdot \mathbf{r})$ \\
Vortex & $\Delta_{1}(\mathbf{r})=\Delta\left(e^{-i \mathbf{q} \cdot \mathbf{r}}+\delta e^{i \mathbf{q} \cdot \mathbf{r}}\right)$ & $\Delta_{1}(\mathbf{r})=\Delta\left(e^{-i \mathbf{q} \cdot \mathbf{r}}+\delta e^{i \mathbf{q} \cdot \mathbf{r}}\right)$ \\
& $\Delta_{2}(\mathbf{r})=\Delta\left(\delta e^{-i \mathbf{q} \cdot \mathbf{r}}+e^{i \mathbf{q} \cdot \mathbf{r}}\right)$ & $\Delta_{2}(\mathbf{r})=\Delta^{\prime} \cos (\mathbf{q} \cdot \mathbf{r})$ \\
& & $\Delta_{3}(\mathbf{r})=\Delta\left(\delta e^{-i \mathbf{q} \cdot \mathbf{r}}+e^{i \mathbf{q} \cdot \mathbf{r}}\right)$ \\
CS & $\Delta_{1}(\mathbf{r})=\Delta\left(e^{i \mathbf{q} \cdot \mathbf{r}}+\delta e^{-i \mathbf{q} \cdot \mathbf{r}}\right)$ & $\Delta_{1}(\mathbf{r})=\Delta\left(e^{i \mathbf{q} \cdot \mathbf{r}}+\delta e^{-i \mathbf{q} \cdot \mathbf{r}}\right)$ \\
& $\Delta_{2}(\mathbf{r})=\Delta\left(\delta e^{i \mathbf{q} \cdot \mathbf{r}}+e^{-i \mathbf{q} \cdot \mathbf{r}}\right)$ & $\Delta_{2}(\mathbf{r})=\Delta^{\prime} \cos (\mathbf{q} \cdot \mathbf{r})$ \\
& & $\Delta_{3}(\mathbf{r})=\Delta\left(\delta e^{i \mathbf{q} \cdot \mathbf{r}}+e^{-i \mathbf{q} \cdot \mathbf{r}}\right)$ \\
\hline \hline
\end{tabular}

\section{BINARY SUPERLATTICE}

\section{A. Bilayer system}

In this section we study the binary superlattices [see Fig. 1(a)], which have been fabricated in $\mathrm{CeCoIn}_{5} / \mathrm{YbCoIn}_{5}$ [25]. We begin with the simplest case, namely the bilayer system $(M=2)$ illustrated in Fig. 2. Then, the strength of the orbital effect is controlled by the Fermi energy $E_{\mathrm{F}}$, which is proportional to the in-plane hopping $t$. The orbital effect is estimated from the dimensionless quantity $H \xi c / \Phi_{0}$, with $\Phi_{0}=\frac{h}{2 e}$ being the flux quantum. Since the coherence length of superconductivity is $\xi \simeq \hbar v_{\mathrm{F}} / k_{\mathrm{B}} T_{\mathrm{c}} \sim\left(E_{\mathrm{F}} / k_{\mathrm{B}} T_{\mathrm{c}}\right) a$, the orbital effect is enhanced by increasing the Fermi energy. On the other hand, the paramagnetic effect of the magnetic field is estimated from another dimensionless quantity $\mu_{\mathrm{B}} H / k_{\mathrm{B}} T_{\mathrm{c}}$, which is independent of the Fermi energy. Thus, the orbital effect (paramagnetic effect and RSOC) plays an important role in the superconducting state for large (small) in-plane hopping $t$. Note that the RSOC induces the CS state through the paramagnetic effect.

When we assume small in-plane hopping $t=20 \mathrm{meV}$ consistent with the heavy effective mass of $\mathrm{CeCoIn}_{5}$, the orbital effect is negligible. Indeed, we obtain the phase diagram in Fig. 3(a), which resembles the result in the paramagnetic limit [31]. The CS state is stable in the high magnetic field region, but the PDW state is not stabilized. Upon increasing the magnetic field, the center-of-mass momentum of Cooper pairs gradually increases through the BCS-CS phase transition [Fig. 3(b)], indicating the second-order phase transition. Figure 3(c) shows that $\Delta_{1}=\Delta_{2}$ in the BCS state while $\delta$ in Eqs. (8) and (9) decreases upon increasing the magnetic field in the CS state. These behaviors are consistent with the previous study on the same model [31] where the orbital effect is simply neglected. Thus, the previous study that was focused on the heavy-fermion superlattice $\mathrm{CeCoIn}_{5} / \mathrm{YbCoIn}_{5}$ is justified.

On the other hand, the orbital effect significantly affects the superconducting state for large in-plane hopping, $t=$ $200 \mathrm{meV}$. Figure 4(a) shows that the vortex state is stable in the high magnetic field region, as expected. The BCS-vortex phase transition is second order as indicated by the continuous change of the center-of-mass momentum [Fig. 4(b)] and $\delta$ [Fig. 4(c)]. Although the RSOC and the paramagnetic effect play less important roles than the orbital effect, they induce a characteristic magnetic field dependence in the center-ofmass momentum around the Pauli-Chandrasekhar-Clogston limit $\mu_{\mathrm{B}} H / k_{\mathrm{B}} T_{\mathrm{c} 0}=1.25$. The RSOC and paramagnetic effect suppress the orbital effect and thus decrease the center-of-mass momentum above the Pauli-Chandrasekhar-Clogston limit.

A main result of our study is obtained when the RSOC competes with the orbital effect. Such a situation is realized for moderate in-plane hopping $t=80 \mathrm{meV}$. Then the PDW state is stabilized in the high magnetic field region, as shown in Fig. 5. Note that the PDW state is induced neither by the orbital effect nor by the paramagnetic effect and RSOC. The PDW state is stable due to a balance of these effects. We explain this mechanism in detail here. The order parameter of the CS and vortex states is described by Eqs. (8) and (9), and these two states are differentiated by the sign of $q$. The positive $q$ (vortex state) is favored by the orbital effect, although the negative $q$ (CS state) is induced by the RSOC. Thus, $q \sim 0$ 


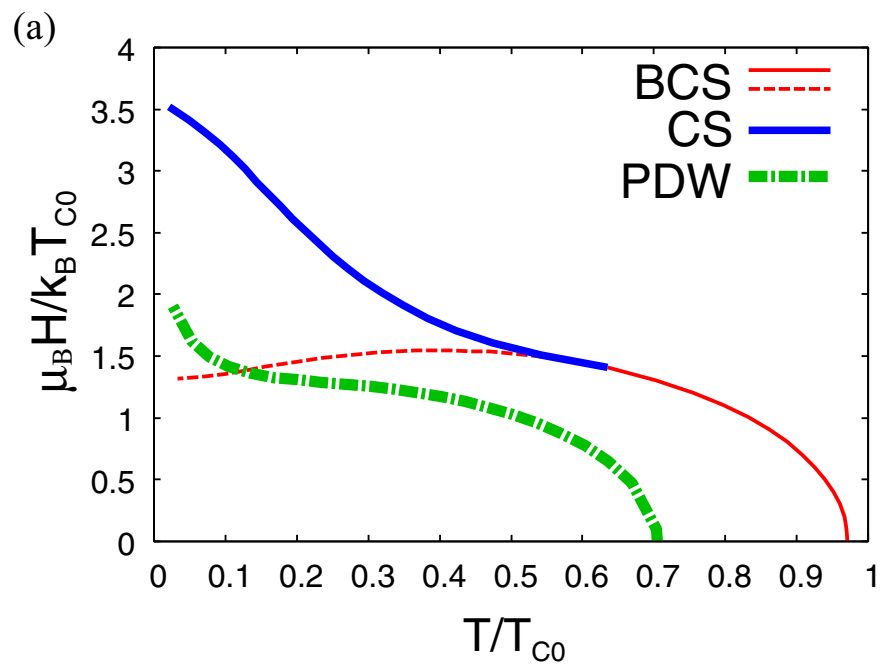

(b)

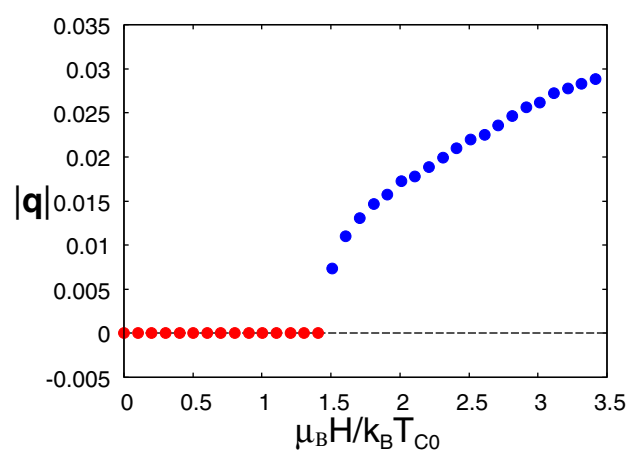

(c)

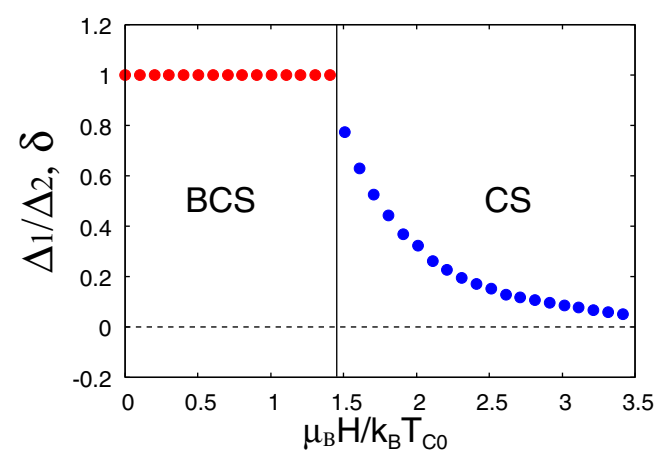

FIG. 3. (Color online) (a) Transition temperatures of various superconducting states in the bilayer system. We assume small in-plane hopping, $t=20 \mathrm{meV}$. Thin (red), moderate (blue), and thick (green) lines show the $T_{\mathrm{c}}$ of BCS, CS, and PDW states, respectively. The highest transition temperature is observable and indicated by the solid line, while the dashed and dot-dashed lines show the fictitious transition temperatures. (b) Magnetic field dependence of the center-of-mass momentum $|\mathbf{q}|$. (c) Layer dependence of the order parameter. We show $\Delta_{2} / \Delta_{1}$ for the BCS state and $\delta$ for the CS state. In this subsection, we choose the pairing interaction $V_{\mathrm{s}} / t=1.5$, which gives the transition temperature $k_{\mathrm{B}} T_{\mathrm{c} 0}=0.0124 t$ in the absence of the spin-orbit coupling and magnetic field. The temperature and magnetic field are scaled by $T_{\mathrm{c} 0}$ in the figures.

when these two effects are in balance. Then, the interlayer Josephson coupling stabilizes the uniform superconducting state along the conducting plane, and thus $q=0$. The $\pi$ phase difference between layers is favored so that the paramagnetic depairing effect is avoided as in the $c$-axis magnetic field [23]. In this way, the PDW state is stabilized by the orbital effect, the paramagnetic effect, RSOC, and interlayer coupling.

\section{B. More-than-two-layer system}

Although it is hard to experimentally control the Fermi energy, the number of superconducting layers $M$ can be tuned by using the artificial superlattice $[25,28,29]$. Thus, we may be able to control the orbital effect by tuning $M$. Since the shift of momentum on the outermost layers, $\left|p_{1}\right|=$ $\left|p_{M}\right|=e H c(M-1) / 2 \hbar$, increases with $M$, the orbital effect is enhanced by increasing the number of superconducting layers. This is reasonable because vortices easily penetrate inside of thick SCs. We demonstrate here that the competing region of the RSOC and the orbital effect is realized by tuning $M$, and then the PDW state is stabilized.

We take into account the RSOC on the outermost layers, $\left(\alpha_{1}, \alpha_{M}\right)=(\alpha,-\alpha)$, while the RSOC on the other layers is neglected for simplicity. This is a reasonable assumption for the layer dependence of RSOC because the spin-orbit coupling is determined by the local environment of atoms [39], and thus the outermost layers contain the largest spin-orbit coupling.

We fix the in-plane hopping, $t=21 \mathrm{meV}$, and the pairing interaction, $V_{\mathrm{s}} / t=1.3$. Then, the orbital effect is negligible in the bilayer system as in Fig. 3. The trilayer system shows the phase diagram (Fig. 6) similar to Fig. 3, and thus the trilayer system is still close to the Pauli limit.

On the other hand, the orbital effect plays an important role in the five-layer system $(M=5)$. Then, the orbital effect competes with the RSOC, and therefore the PDW state is stabilized as expected from the results in Sec. III A. Indeed, Fig. 7 shows that the BCS state, the CS state, and the PDW state are stabilized in the low, intermediate, and high magnetic field regions, respectively. Because the CS-PDW transition is a first- 
(a)

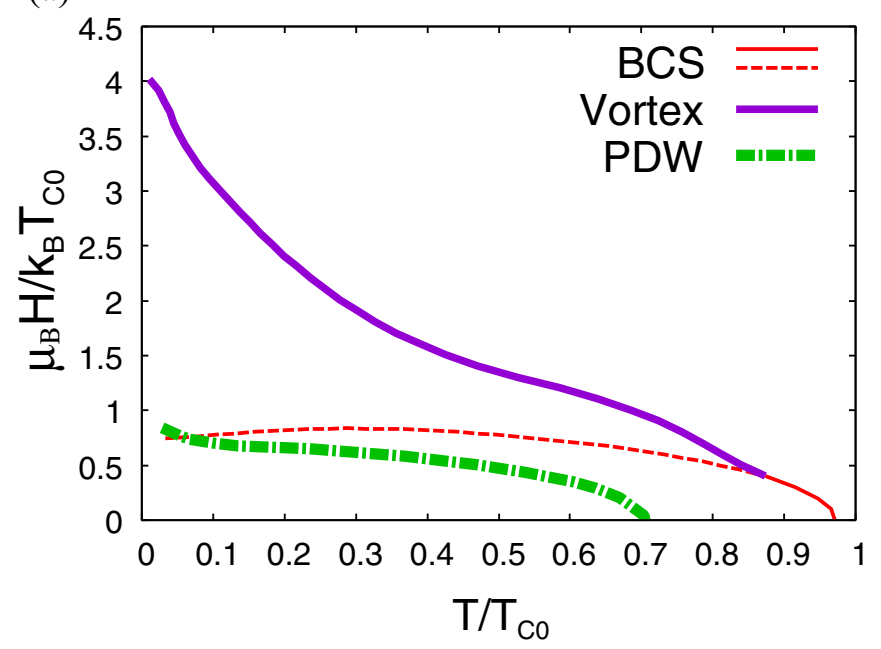

(b)

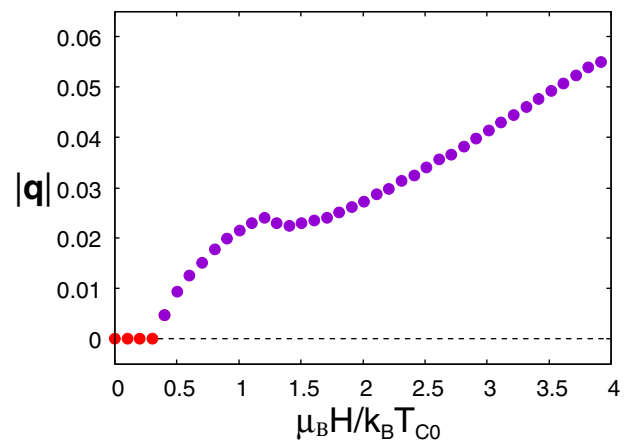

(c)

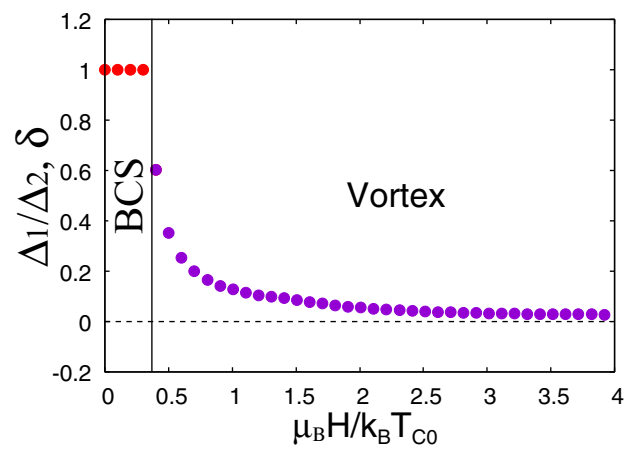

FIG. 4. (Color online) Transition temperatures (a), center-of-mass momentum (b), and layer-dependent order parameter (c) for a large in-plane hopping, $t=200 \mathrm{meV}$. The other parameters are the same as those in Fig. 3. The moderate (purple) line in (a) shows the transition temperature of the vortex state.

order phase transition, the upper critical field shows a kink, although the kink may be weak in some cases [see Figs. 5(a) and 10(a)]. Generally speaking, a distinct kink appears when the transition temperature of the PDW state is small. Then, the $H_{\mathrm{c} 2}$ of the CS state is suppressed while that of the PDW state shows an upward curvature. The observation of the kink will be an experimental test for the presence of the PDW state.

When we furthermore increase the number of superconducting layers, the superconducting state is dominated by the orbital effect, and thus the vortex state is stabilized in the high magnetic field region. For example, we show the phase diagram of the seven-layer system $(M=7)$ in Fig. 8. It is shown that the PDW state is not stabilized.

We focus here on the five-layer system, and we emphasize the cooperative role of the RSOC, the orbital effect, and the paramagnetic effect on the thermodynamic stability of the PDW state. Figure 9(a) shows the phase diagram in the absence of the orbital effect. The CS state is more stable than the PDW state, as in the bilayer and trilayer systems. On the other hand, the vortex state is stable in the high magnetic field region when we neglect the paramagnetic effect, as shown in Fig. 9(b). Note that the RSOC does not play an important role in the absence of the paramagnetic effect.
It should be noticed that the orbital limit of the upper critical field [Fig. 9(b)] is much larger than the paramagnetic limit [see Fig. 9(a)], indicating the large Maki parameter. This means that the upper critical field of five-layer systems is mainly determined from the paramagnetic effect. In this sense, the PDW state occurs near the paramagnetic limit, although a weak orbital effect is needed. Stars in Figs. 6, 7, 8, and 9(a) show the crossover induced by the paramagnetic effect [23]. Because the center layer is not protected against the paramagnetic effect by the RSOC, the order parameter in the center layer $\Delta_{(M+1) / 2}$ suddenly decreases by increasing the magnetic field through the crossover. We see that the paramagnetic effect appears even in the seven-layer system, where the orbital effect is larger than the effect of RSOC.

\section{C. $\mathrm{CeCoIn}_{5} / \mathrm{YbCoIn}_{5}$}

In the previous subsections, we designed the odd-parity PDW state using the artificial heterostructures. It has been shown that the orbital effect is controlled by the number of superconducting layers. Indeed, various superlattices CeCoIn $5 / \mathrm{YbCoIn}_{5}$ with $M>2$ are superconducting, and we can tune the number of layers $M[25,28,29]$. Thus, the artificial superlattice $\mathrm{CeCoIn}_{5} / \mathrm{YbCoIn}_{5}$ may be a new platform for odd- 
(a)
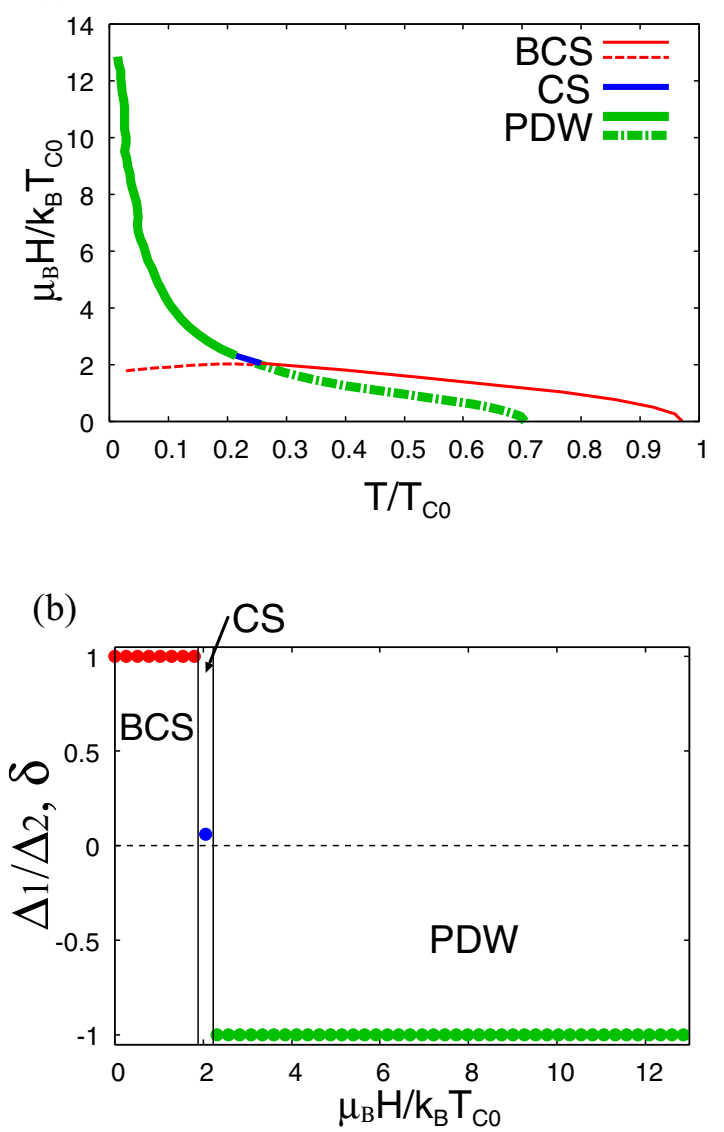

FIG. 5. (Color online) Transition temperatures (a) and layer dependence of the order parameter (b) for a moderate in-plane hopping, $t=80 \mathrm{meV}$. The other parameters are the same as those in Fig. 3. It is shown that the PDW state is stable in the high magnetic field region.

parity superconductivity. A reasonable parameter $t \sim 20 \mathrm{meV}$ leads to the PDW state in the five-layer system.

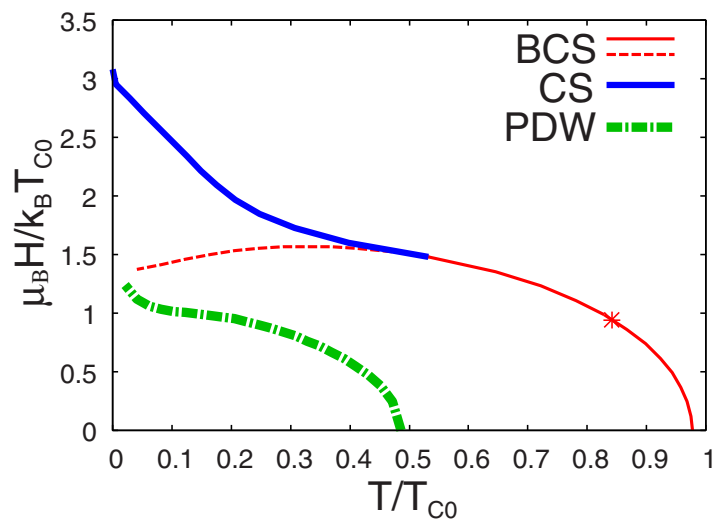

FIG. 6. (Color online) Transition temperatures of the BCS, CS, and PDW states in the trilayer system $(M=3)$ for small in-plane hopping, $t=21 \mathrm{meV}$. In this subsection, we assume $V_{\mathrm{s}} / t=1.3$, which gives rise to the transition temperature at zero magnetic field, $T_{\mathrm{c} 0}=0.00487 t / k_{\mathrm{B}}$, in the absence of RSOC.

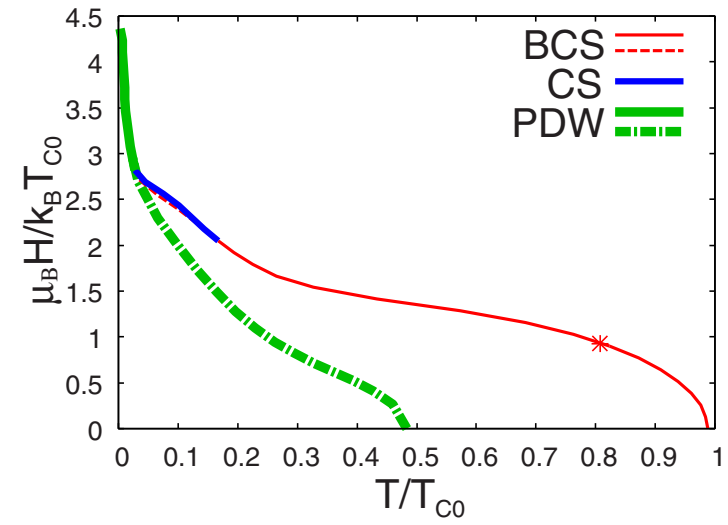

FIG. 7. (Color online) Transition temperatures of the BCS, CS, and PDW states in the five-layer system $(M=5)$ for a small in-plane hopping, $t=21 \mathrm{meV}$.

We comment here on the recent experimental observations of the paramagnetic effect in the superlattice $\mathrm{CeCoIn}_{5} / \mathrm{YbCoIn}_{5}$ [28]. Goh et al. observed the strong paramagnetic effect by measuring the field-angle dependence of the upper critical field. They also showed that the paramagnetic effect is suppressed in a few-layer system $M \leqslant 3$. Their experimental results are consistent with our model; the paramagnetic effect is suppressed with decreasing $M$ because the superconductivity in surface layers, $m=1$ and $M$, is substantially protected against the paramagnetic effect due to the RSOC $[20,28]$. Indeed, the upper critical field of the trilayer system is larger than that of the five-layer system near $T=T_{\mathrm{c} 0}$ (see Figs. 6 and 7). Note that the upper critical field is dominantly determined from the paramagnetic effect even when the orbital effect competes with the RSOC (see the discussion in Sec. III B).

Considering the consistency between our calculation and experiments for $\mathrm{CeCoIn}_{5} / \mathrm{YbCoIn}_{5}$ at low magnetic fields, it is expected that the PDW state may be realized in the artificial superlattice with $M \simeq 5$ at high magnetic fields. However, any indication of the presence of a high-field superconducting phase has not been reported. For instance, the kink and the upturn of the upper critical field shown in our calculations have

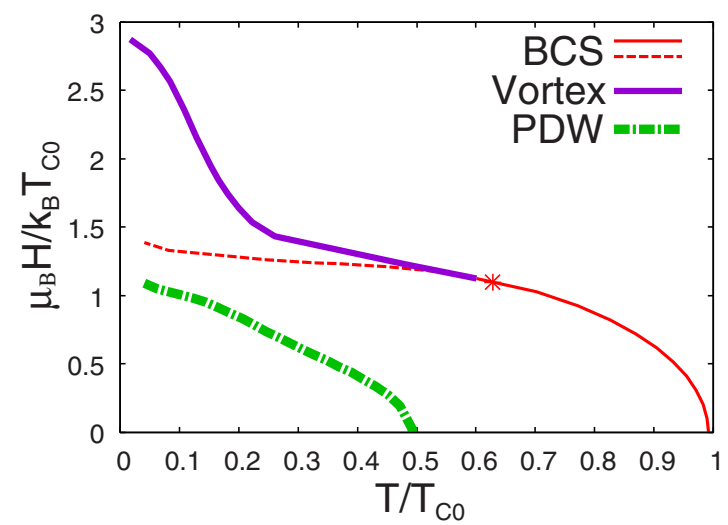

FIG. 8. (Color online) Transition temperatures of the BCS, the vortex, and the PDW states in the seven-layer system $(M=7)$ for small in-plane hopping, $t=21 \mathrm{meV}$. 
(a)

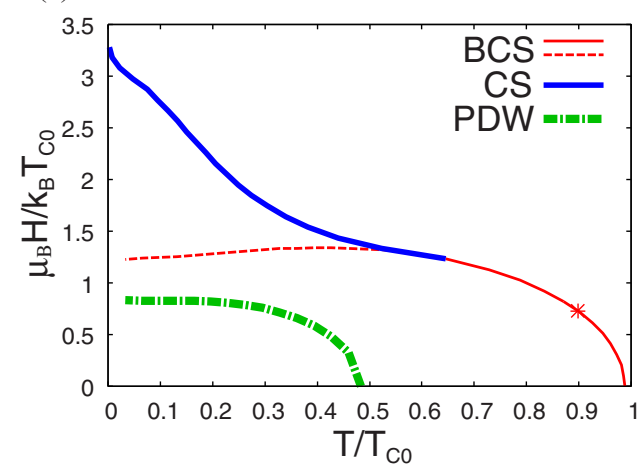

(b)

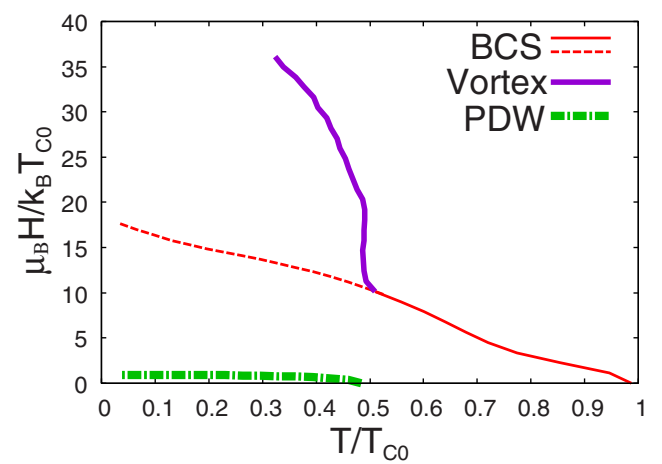

FIG. 9. (Color online) Transition temperatures of the superconducting states in the five-layer system. (a) Pauli limit by setting $\boldsymbol{p}_{m}=0$. (b) Orbital limit by eliminating the Zeeman term in the Hamiltonian [Eq. (1)]. The other parameters are the same as those in Fig. 7.

not been observed [25,28,29]. The high-field phase may have been missed because the measurement has not been carried out in the low-temperature region. On the other hand, the discrepancy may be attributed to the two ingredients that are not taken into account in our model. First, it is expected that disorders suppress the high-field superconducting phase, as the FFLO state is suppressed [40]. The artificial superlattice $\mathrm{CeCoIn}_{5} / \mathrm{YbCoIn}_{5}$ indeed contains substantial disorders. Second, the number of spacer layers $N=4-6$ may not be large enough to eliminate the coupling between superconducting multilayers. Intermultilayer coupling is harmful for the PDW state, and thus it should be decreased by increasing the number of spacer layers.

\section{TRICOLOR SUPERLATTICE}

Next, we discuss the tricolor superlattice illustrated in Fig. 1(b). As we have shown in Sec. III B, the orbital effect is controlled by the spacing between the outermost layers. Thus, the PDW state may be stabilized in a tricolor superlattice by intercalating the spacer layers into the superconducting layers. Then, the spacing of neighboring superconducting layers is multiplied to $d=\left(m_{\mathrm{d}}+1\right) c$, with $m_{\mathrm{d}}$ being the number of intercalated spacer layers [green open circles in Fig. 1(b)]. We assume here that the interlayer spacing between the spacer layer and the superconducting layer is c. A similar situation in the bilayer $\delta$-doped $\mathrm{SrTiO}_{3}$ [41] has been realized, and superconductivity in the bilayer interface $\mathrm{LaAlO}_{3} / \mathrm{SrTiO}_{3} / \mathrm{LaAlO}_{3}$ has been studied [22].

Multiplying the interlayer spacing by $\left(m_{\mathrm{d}}+1\right)$ is equivalent to increasing the in-plane hopping to $\left(m_{\mathrm{d}}+1\right) t$ while keeping the ratio, $t_{\perp} / t, \mu / t, \alpha / t$, and $V_{\mathrm{s}} / t$. For instance, we obtain the same results for the binary superlattice with $t=80 \mathrm{meV}$ and for the tricolor superlattice with $t=20 \mathrm{meV}$ and $m_{\mathrm{d}}=3$. Thus, the PDW state may be stabilized in the tricolor bilayer superlattice. However, in reality, the interlayer hopping $t_{\perp}$ between the nearest-neighbor superconducting layers is significantly decreased by intercalating a spacer layer. For instance, we obtain $t_{\perp} \sim t_{\mathrm{ns}}^{2} /\left(E_{\mathrm{s}}-E_{\mathrm{n}}\right)$ in the presence of a single spacer layer $\left(m_{\mathrm{d}}=1\right)$, where $t_{\mathrm{ns}}$ is the hopping integral between the superconducting layer and the spacer layer and $E_{\mathrm{s}}-E_{\mathrm{n}}$ is the potential difference between these layers. Therefore, the stability of the PDW state in the tricolor superlattice should be examined by investigating the superconducting state for small $t_{\perp}$.

We study here the tricolor bilayer superlattice for simplicity. The in-plane hopping is set to $t=20 \mathrm{meV}$ by considering the heavy-fermion superlattice. As we discussed above, we obtain the same phase diagram as Fig. 5 when $m_{\mathrm{d}}=3$ and $t_{\perp} / t=0.1$. On the other hand, Figs. 10(a) and 10(b) show the phase diagram for $t_{\perp} / t=0.05$ and 0.01 , respectively. Since the

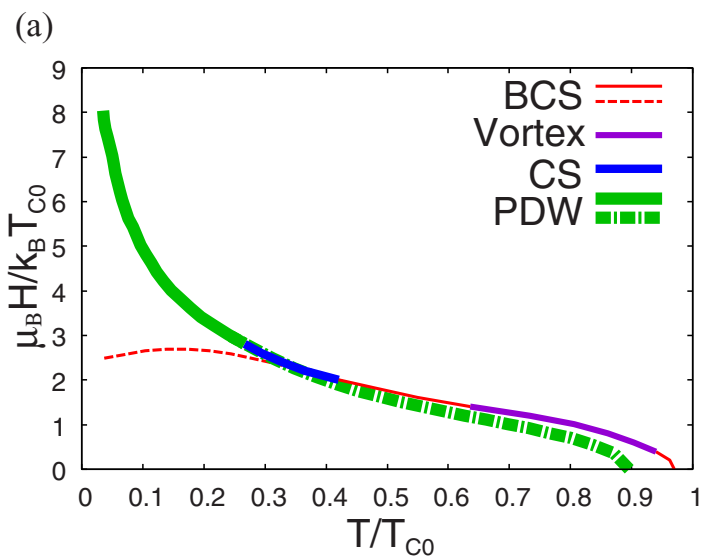

(b)

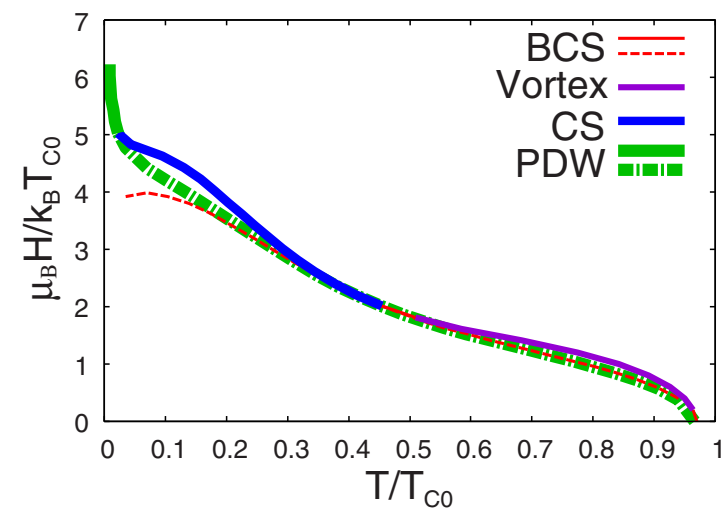

FIG. 10. (Color online) Transition temperatures of the BCS, CS, vortex, and PDW states in the tricolor superlattice with $m_{\mathrm{d}}=3, t=$ $20 \mathrm{meV}, \mu / t=2, \alpha / t=0.3$, and $V_{\mathrm{s}} / t=1.5$. (a) $t_{\perp} / t=0.05$ and (b) $t_{\perp} / t=0.01$. The temperature is normalized by $T_{\mathrm{c} 0}=0.0124 t / k_{\mathrm{B}}$. 
interlayer Josephson coupling decreases with $t_{\perp} / t$, the uniform states, namely the BCS and PDW states, are suppressed. It is shown that the PDW state is stable at high magnetic fields, but the transition temperature of the PDW state is significantly decreased for $t_{\perp} / t=0.01$. The PDW state will be furthermore suppressed by further decreasing $t_{\perp} / t$. Thus, it may be hard to realize the PDW state in a tricolor superlattice by intercalating many spacer layers.

It should be noticed that Fig. 10(b) is similar to the phase diagram of the binary five-layer superlattice (Fig. 7). We now understand that the reduced transition temperature of the PDW state in the five-layer system is due to the reduced Josephson coupling between the outermost layers. In other words, the superconducting inner layers play a role of the spacer layers. Indeed, the PDW state is mainly induced by the outermost layers where the superconductivity is protected against the paramagnetic effect by the RSOC. For example, we obtain the layer-dependent order parameter $\left(\Delta_{1}, \Delta_{2}, \Delta_{3}, \Delta_{4}, \Delta_{5}\right) \simeq$ $\Delta(1,0.18,0,-0.18,-1)$ in the five-layer PDW state.

Finally, we discuss the superconductivity in the bilayer $\delta$-doped $\mathrm{SrTiO}_{3}$ [41] and the bilayer interface $\mathrm{LaAlO}_{3} / \mathrm{SrTiO}_{3} / \mathrm{LaAlO}_{3}$ [22]. The Fermi velocity estimated for a carrier density $n \sim 10^{14} \mathrm{~cm}^{-2}$ on the basis of the three-orbital tight-binding model [42] is approximately twice as large as that in our model. Since the lattice constant along the $c$ axis, $c=3.9 \AA$, is nearly half of $\mathrm{CeCoIn}_{5}$, the orbital effect in the $\mathrm{SrTiO}_{3}$ heterostructures is comparable to our model. Thus, the bilayer system sandwiching three nonsuperconducting layers may be a platform of the odd-parity PDW state. Then, the interlayer coupling between superconducting layers may be small, and therefore the PDW state may appear in the low-temperature region, as shown in Fig. 10(b). The kink in the upper critical field would be a signature of the PDW state.

\section{TOPOLOGICAL SUPERCONDUCTIVITY}

Topologically nontrivial insulators and SCs have evolved into one of the major research topics of modern condensedmatter physics recently $[43,44]$. In particular, topological superconductivity attracts a great deal of attention since the Majorana state satisfying the non-Abelian statistics appears at the edge and dislocations [10-12]. In addition to the "strong" topological phases classified based on the topological Periodic Table $[13,14]$, theories on the symmetry-protected topological superconducting phases (topological crystalline superconductivity) have developed recently [45-49]. In the $c$-axis magnetic field, the odd-parity PDW state is a topological crystalline SC protected by mirror symmetry [24]. The mirror symmetry along the $a b$ plane protects the topological invariant, that is, the mirror Chern number [50] of symmetry class $D$. We obtain the finite mirror Chern number $v( \pm i)=\mp 1$, marking the topologically nontrivial properties of superconductivity, when the number of superconducting layers $M$ is odd [24]. On the other hand, the mirror Chern number is no longer a topological invariant when the magnetic field is tilted from the $c$ axis.

In this section, we show that the PDW state may belong to another kind of topological crystalline SC when the magnetic field is applied along the $a$ or $b$ axis. We demonstrate the topologically nontrivial properties on the basis of the $\mathrm{BdG}$
Hamiltonian,

$$
\begin{aligned}
\mathcal{H}_{\mathrm{BdG}}= & \sum_{\boldsymbol{k}, s, m} \xi\left(\boldsymbol{k}+\boldsymbol{p}_{m}\right) c_{\boldsymbol{k} s m}^{\dagger} c_{\boldsymbol{k s} m}+t_{\perp} \sum_{\boldsymbol{k}, s,\left\langle m, m^{\prime}\right\rangle} c_{\boldsymbol{k} s m}^{\dagger} c_{\boldsymbol{k s} m^{\prime}} \\
& -\sum_{\boldsymbol{k}, s, s^{\prime}, m} \mu_{\mathrm{B}} \boldsymbol{H} \cdot \boldsymbol{\sigma}_{s s^{\prime}} c_{\boldsymbol{k} s m}^{\dagger} c_{\boldsymbol{k} s^{\prime} m} \\
& +\sum_{\boldsymbol{k}, s, s^{\prime}, m} \alpha_{m} \boldsymbol{g}\left(\boldsymbol{k}+\boldsymbol{p}_{m}\right) \cdot \boldsymbol{\sigma}_{s s^{\prime}} c_{\boldsymbol{k} s m}^{\dagger} c_{\boldsymbol{k} s^{\prime} m} \\
& +\frac{1}{2} \sum_{\boldsymbol{k}, s, s^{\prime}, m}\left[\Delta_{s s^{\prime} m}(\boldsymbol{k}) c_{\boldsymbol{k} s m}^{\dagger} c_{-\boldsymbol{k} s^{\prime} m}^{\dagger}+\text { H.c }\right]
\end{aligned}
$$

where $\hat{\Delta}_{m}(\boldsymbol{k}) \equiv\left[\Delta_{s s^{\prime} m}(\boldsymbol{k})\right]=\left[\psi_{m}+\boldsymbol{d}_{m}(\boldsymbol{k}) \cdot \boldsymbol{\sigma}\right] i \sigma_{y}$ describes the layer-dependent order parameter of superconductivity [1]. Although the purely $s$-wave superconductivity is considered in Secs. III and IV, the $p$-wave component is admixed through the layer-dependent RSOC by the local violation of inversion symmetry [51]. The layer dependence of the order parameter is obtained as

$$
\begin{array}{r}
\left(\psi_{1}, \psi_{2}, \psi_{3}, \psi_{4}, \psi_{5}\right)=\left(\psi_{\mathrm{out}}, \psi_{\mathrm{in}}, 0,-\psi_{\mathrm{in}},-\psi_{\mathrm{out}}\right), \quad(11) \\
\left(\boldsymbol{d}_{1}(\boldsymbol{k}), \boldsymbol{d}_{2}(\boldsymbol{k}), \boldsymbol{d}_{3}(\boldsymbol{k}), \boldsymbol{d}_{4}(\boldsymbol{k}), \boldsymbol{d}_{5}(\boldsymbol{k})\right)=\left(d_{\mathrm{out}}, d_{\mathrm{in}}, d_{\mathrm{in}}^{\prime}, d_{\mathrm{in}}, d_{\mathrm{out}}\right) \boldsymbol{g}(\boldsymbol{k})
\end{array}
$$

in the five-layer PDW state. The BdG Hamiltonian is represented in Nambu space,

$$
\begin{gathered}
\mathcal{H}_{\mathrm{BdG}}=\frac{1}{2} \sum_{\boldsymbol{k}} \hat{c}^{\dagger} \hat{H}_{\mathrm{BdG}}(\boldsymbol{k}) \hat{c}, \\
\hat{H}_{\mathrm{BdG}}(\boldsymbol{k})=\left(\begin{array}{cc}
\hat{H}_{0}(\boldsymbol{k}) & \hat{\Delta}(\boldsymbol{k}) \\
\hat{\Delta}^{\dagger}(\boldsymbol{k}) & -\hat{H}_{0}(-\boldsymbol{k})^{T}
\end{array}\right),
\end{gathered}
$$

where $\hat{H}_{0}(\boldsymbol{k})$ is the Hamiltonian in the normal state and $\hat{\Delta}(\boldsymbol{k})$ is the order parameter.

Although the mirror symmetry with respect to the $a b$ plane $\mathcal{M}_{a b}$ is broken in the in-plane magnetic field, the magnetic mirror symmetry $\mathcal{T}^{\prime}=\mathcal{T} M_{c a}\left(\mathcal{T} M_{b c}\right)$ is preserved in the $a$-axis ( $b$-axis) magnetic field. For instance, in the magnetic field along the $a$ axis, the BdG Hamiltonian is invariant under magnetic mirror symmetry,

$$
\mathcal{T}^{\prime} \hat{H}_{\mathrm{BdG}}\left(k_{x}, k_{y}\right) \mathcal{T}^{\prime \dagger}=\hat{H}_{\mathrm{BdG}}\left(-k_{x}, k_{y}\right),
$$

where $\mathcal{M}_{c a}=i \sigma_{y}$ is the mirror reflection operator and $\mathcal{T}=i \sigma_{y} \mathcal{K}$ is the time-reversal operator, with $\mathcal{K}$ being the complex-conjugate operator. Combining with the particle-hole symmetry $\mathcal{C} \hat{H}_{\mathrm{BdG}}(\boldsymbol{k}) \mathcal{C}^{\dagger}=-\hat{H}_{\mathrm{BdG}}(-\boldsymbol{k})$, where $\mathcal{C}=\tau_{x} \mathcal{K}$ and $\tau_{x}$ is the Pauli matrix in the particle-hole space, we can define the mirror chiral symmetry $\Gamma \hat{H}_{\mathrm{BdG}}\left(k_{x}, k_{y}\right) \Gamma^{\dagger}=-\hat{H}_{\mathrm{BdG}}\left(k_{x},-k_{y}\right)$ with $\Gamma=-\mathcal{C} T^{\prime}=\tau_{x}$. Thus, the BdG Hamiltonian satisfies the chiral symmetry

$$
\left\{\Gamma, \hat{H}_{\mathrm{BdG}}(\boldsymbol{k})\right\}=0
$$

at $k_{y}=0$ and $k_{y}=\pi / a$. The chiral symmetry ensures that the one-dimensional winding number

$$
\omega_{k_{y}}=\frac{1}{4 \pi i} \int_{-\frac{\pi}{a}}^{\frac{\pi}{a}} d k_{x} \operatorname{Tr}\left[\hat{q}(\boldsymbol{k})^{-1} \partial_{k_{x}} \hat{q}(\boldsymbol{k})-\hat{q}^{\dagger}(\boldsymbol{k})^{-1} \partial_{k_{x}} \hat{q}^{\dagger}(\boldsymbol{k})\right]
$$


is a topological invariant [52-57] when a finite gap is open at $k_{y}=0$ and $k_{y}=\pi / a$. The $2 M \times 2 M$ matrix $\hat{q}(\boldsymbol{k})$ is obtained by carrying out the unitary transformation

$$
U \hat{H}_{\mathrm{BdG}}(\boldsymbol{k}) U^{\dagger}=\left(\begin{array}{cc}
0 & \hat{q}(\boldsymbol{k}) \\
\hat{q}^{\dagger}(\boldsymbol{k}) & 0
\end{array}\right) .
$$

When we regard the magnetic mirror symmetry $\mathcal{T}^{\prime}$ as pseudo-time-reversal symmetry [58,59], the one-dimensional Hamiltonian $\hat{H}_{1 \mathrm{D}}^{k_{y}=0}\left(k_{x}\right)=\hat{H}_{\mathrm{BdG}}\left(k_{x}, 0\right)$ and $\hat{H}_{1 \mathrm{D}}^{k_{y}=\pi / a}\left(k_{x}\right)=$ $\hat{H}_{\mathrm{BdG}}\left(k_{x}, \pi / a\right)$ belong to the symmetry class $B D I$ because $\mathcal{T}^{\prime 2}=+1[13,14]$. Thus, we can define the integer topological numbers of the $B D I$ class,

$$
\begin{gathered}
v_{0}^{B D I}=\frac{1}{\pi i} \int_{0}^{\frac{\pi}{a}} d k_{x} \operatorname{Tr}\left[\hat{q}\left(k_{x}, 0\right)^{-1} \partial_{k_{x}} \hat{q}\left(k_{x}, 0\right)\right], \\
v_{\pi / a}^{B D I}=\frac{1}{\pi i} \int_{0}^{\frac{\pi}{a}} d k_{x} \operatorname{Tr}\left[\hat{q}\left(k_{x}, \pi / a\right)^{-1} \partial_{k_{x}} \hat{q}\left(k_{x}, \pi / a\right)\right] .
\end{gathered}
$$

Indeed, these winding numbers are equivalent to Eq. (17), namely $v_{0, \pi / a}^{B D I}=\omega_{0, \pi / a}$. The pseudo-time-reversal symmetry considered here has been used for the definition of the integer topological number in one-dimensional semiconductor nanowires [58] and quasi-one-dimensional $d$-wave superconductors [59]. The magnetic mirror symmetry is the physical origin of this "hidden" time-reversal symmetry. The difference of two winding numbers, $v_{0}^{B D I}-v_{\pi / a}^{B D I}$, is the strong index of $2 \mathrm{D}$ topological crystalline SCs protected by the magnetic mirror symmetry [48].

We now discuss the superconducting gap. Figure 11 shows the gap of the single-particle excitation spectra in the five-layer PDW state for each $k_{y}$, which is defined as $E_{\min }\left(k_{y}\right)=$ $\min _{i, k_{x}}\left|E_{i}(\boldsymbol{k})\right|$, with $E_{i}(\boldsymbol{k})$ being eigenvalues of the $\mathrm{BdG}$ Hamiltonian $\hat{H}_{\mathrm{BdG}}(\boldsymbol{k})$. We assume here $\mu / t=-2$ so that the Fermi surface encloses the $\Gamma$ point $(\boldsymbol{k}=\mathbf{0})$. Since there is no

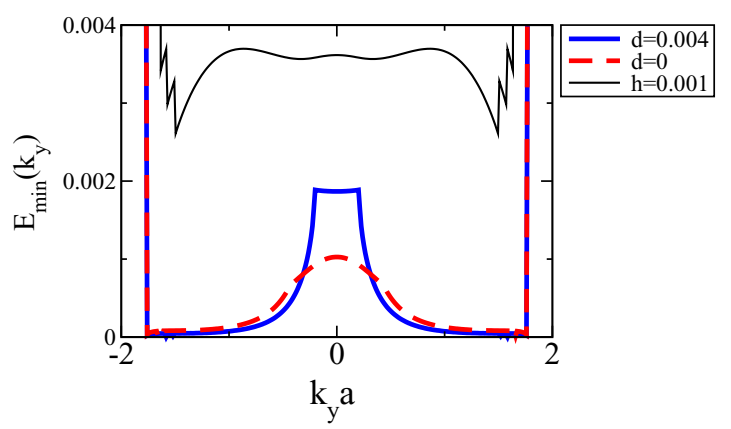

FIG. 11. (Color online) Superconducting gap at each $k_{y}$ in the five-layer PDW state, defined by $E_{\min }\left(k_{y}\right)=\min _{i, k_{x}}\left|E_{i}(\boldsymbol{k})\right|$. We assume $t=20 \mathrm{meV}, t_{\perp} / t=0.1, \mu / t=-2, \alpha / t=0.3$, and $\mu_{\mathrm{B}} H / t=$ 0.04 is the magnetic field along the $a$ axis. The layer-dependent order parameters are chosen as $\psi_{\text {out }}=0.02$ and $\psi_{\text {in }}=0.0036$ consistent with the results in Sec. III B (Fig. 7). We take into account a small $p$-wave component $d \equiv d_{\text {out }}=d_{\text {in }}=d_{\text {in }}^{\prime}=0.004$ induced by the RSOC [51] (thick solid line), while we obtain the dashed line for $d=0$. We also show the gap for $d=0.004$ and $\mu_{\mathrm{B}} H / t=0.001$ for a comparison (thin solid line). The bulk gap opens in the low magnetic field region, but it closes in the high magnetic field region where the PDW state is stable. The superconducting gap at $k_{y}=0$ is finite even in the high magnetic field region.

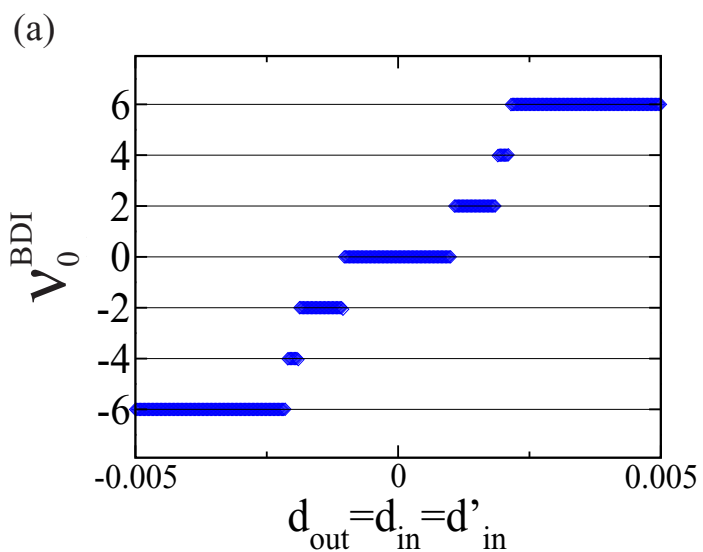

(b)

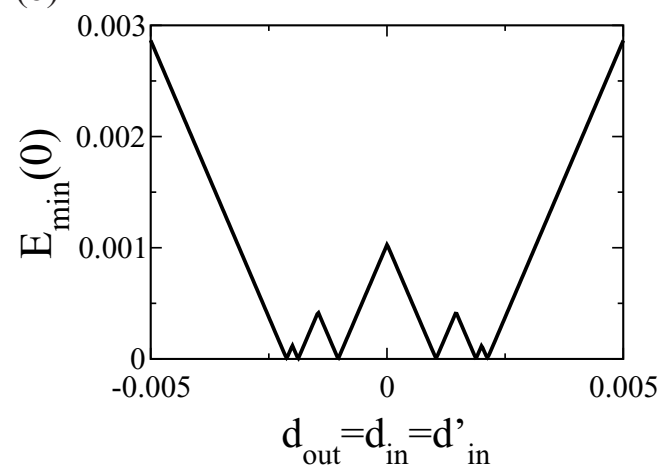

FIG. 12. (Color online) (a) Winding number and (b) superconducting gap at $k_{y}=0$ as a function of the $p$-wave component $d \equiv d_{\text {out }}=d_{\text {in }}=d_{\text {in }}^{\prime}$. The other parameters are the same as those in Fig. 11.

Fermi surface along $k_{y}=\pi / a$, the winding number is trivial, $v_{\pi / a}^{B D I}=0$. Therefore, we focus on $v_{0}^{B D I}$. The superconducting gap is finite at $k_{y}=0$, ensuring the topological protection of the winding number $v_{0}^{B D I}$. At low magnetic fields, the superconducting gap is finite in the whole Brillouin zone (thin solid line in Fig. 11), and thus $v_{0}^{B D I}$ is the strong topological index. Although the gap at finite $k_{y}$ is closed at high magnetic fields due to the paramagnetic effect, the winding number is regarded as a topological number of an effective one-dimensional Hamiltonian.

As we show in Fig. 12(a), $v_{0}^{B D I}$ discretely changes upon increasing the $p$-wave component in the order parameter, $d \equiv$ $d_{\text {out }}=d_{\text {in }}=d_{\text {in }}^{\prime}$. The superconducting gap is closed for special values of $d$ where the winding number jumps [Fig. 12(b)]. We ignore here the layer dependence of the $p$-wave component for simplicity. This assumption has been justified by the BdG equation, which shows the nearly layer-independent $p$-wave component in the PDW state [51].

Switching on a small $p$-wave component $|d| \geqslant 0.05 \psi_{\text {out }}$, we obtain a finite winding number indicating the topologically nontrivial properties [see Fig. 12(a)]. This is in sharp contrast to the $2 \mathrm{D}$ Rashba SC, where the $p$-wave component overwhelming the $s$-wave component is required for topological superconductivity $[52,60]$. This condition is hardly realized in real materials. On the other hand, a small $p$-wave component induced by the RSOC causes the PDW state to be 


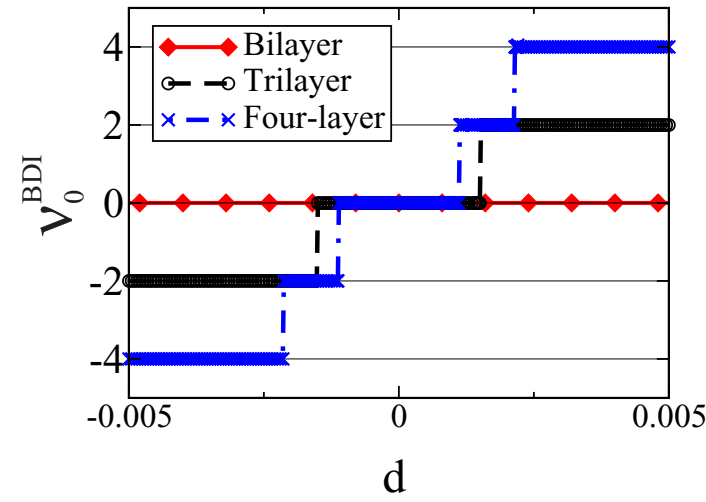

FIG. 13. (Color online) Winding number of the bilayer, trilayer, and four-layer PDW state. We assume a layer-independent $p$-wave component $d \equiv d_{\text {out }}=d_{\text {in }}$ as in Fig. 12. The $s$-wave component has a layer dependence $\left(\psi_{1}, \psi_{2}\right)=\left(\psi_{\text {out }},-\psi_{\text {out }}\right),\left(\psi_{1}, \psi_{2}, \psi_{3}\right)=$ $\left(\psi_{\text {out }}, 0,-\psi_{\text {out }}\right)$, and $\left(\psi_{1}, \psi_{2}, \psi_{3}, \psi_{4}\right)=\left(\psi_{\text {out }}, \psi_{\text {in }},-\psi_{\text {in }},-\psi_{\text {out }}\right)$. The parameters are the same as those in Fig. 11.

topologically nontrivial, because the $s$-wave component of the order parameter is small on inner layers when $M \geqslant 3$. In Fig. 13, we show the winding number of the bilayer, trilayer, and four-layer systems in the PDW state. Although the bilayer system $(M=2)$ is trivial, the topologically nontrivial superconducting state is induced by a small $p$-wave component for $M \geqslant 3$. According to the random-phase-approximation (RPA) analysis of the three-dimensional Rashba-Hubbard model, the induced spin-triplet component is approximately $20 \%$ of the spin-singlet component for a moderate RSOC [39,61]. Thus, the $p$-wave component is likely to be large enough to realize the topological crystalline superconductivity protected by the magnetic mirror symmetry for $M \geqslant 3$. Note that these conditions are different from those for the topological superconductivity in the magnetic field along the $c$ axis. The PDW state is a topological crystalline superconductor in the $c$-axis magnetic field when the number of superconducting layers $M$ is odd [24]. Then, the $p$-wave component is not required.

A nontrivial winding number may ensure the presence of the Majorana edge state according to the bulk-edge correspondence. When the magnetic field is applied along the $a$ axis, the magnetic mirror symmetry $\mathcal{T} M_{c a}$ is preserved at the edge perpendicular to the [100] axis [(100) edge]. Therefore, the winding number protected by this symmetry corresponds to the number of zero-energy edge states according to the index theorem [53]. Indeed, we show the Majorana edge states in Fig. 14. The trilayer PDW state with a large superconducting gap is considered for simplicity of numerical calculation. The energy spectrum is calculated in the open boundary condition along the $a$ axis. The layer-dependent order parameters are $\left(\psi_{1}, \psi_{2}, \psi_{3}\right)=(1,0,-1) \psi_{\text {out }}$ and $\left(\boldsymbol{d}_{1}(\boldsymbol{k}), \boldsymbol{d}_{2}(\boldsymbol{k}), \boldsymbol{d}_{3}(\boldsymbol{k})\right)=$ $(1,1,1) d \boldsymbol{g}(\boldsymbol{k})$. We see the two Majorana modes around $k_{y}=0$ [Fig. 14(a)] when we assume a small $p$-wave component leading to the winding number $\nu_{0}^{B D I}=2$. The Majorana states have a linear dispersion since the chiral symmetry defined in Eq. (16) is not preserved at $0<\left|k_{y}\right|<\pi / a$. Because another pseudo-time-reversal symmetry, $\mathcal{T}^{\prime \prime} \hat{H}_{\mathrm{BdG}}(\boldsymbol{k}) \mathcal{T}^{\prime \prime \dagger}=\hat{H}_{\mathrm{BdG}}(-\boldsymbol{k})$ (a)

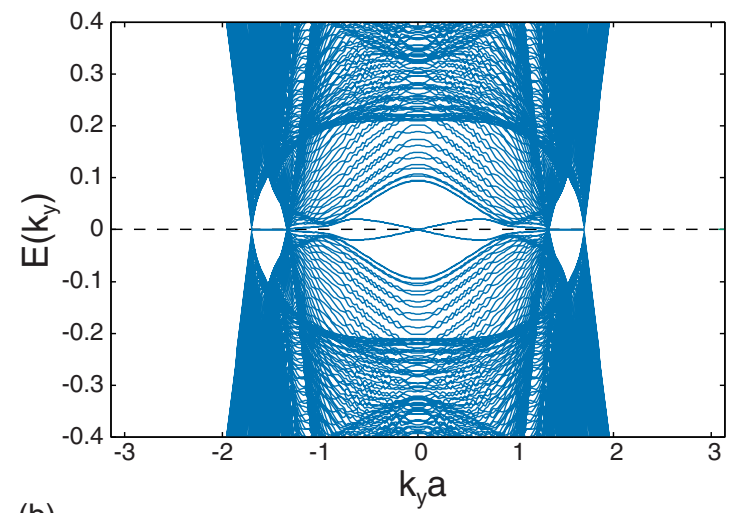

(b)

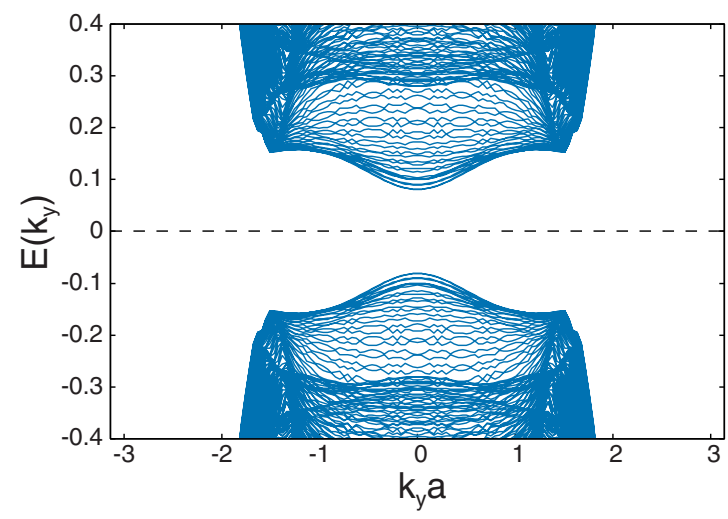

FIG. 14. (Color online) Energy spectra in the trilayer PDW state with open boundaries along the $a$ axis. We consider the magnetic field along the $a$ axis $\mu_{\mathrm{B}} H / t=0.3$ (a) and the $b$ axis $\mu_{\mathrm{B}} H / t=0.1$ (b). We assume $t_{\perp} / t=0.1, \mu / t=-2, \alpha / t=0.3$, and we choose the $s$-wave order parameter $\psi_{\text {out }}=0.5$ and the $p$-wave order parameter $d=0.2$. The orbital effect is neglected for simplicity.

with $\mathcal{T}^{\prime \prime}=\mathcal{T} \mathcal{M}_{a b}$, is preserved, the two Majorana states form "Kramers pairs."

Figure 14(a) also shows the zero-energy flat band at $\left|k_{y} a\right|=$ 1.35-1.7. This mode is specified by another winding number $\omega_{k_{y}}^{\prime}$ protected by the pseudo-time-reversal symmetry $\mathcal{T}^{\prime \prime}$. The winding number $\omega_{k_{y}}^{\prime}$ is defined at all $k_{y}$, and we obtain $\omega_{k_{y}}^{\prime}=$ -1 at $k_{y}$ where the flat band appears. Hence, the zero-energy flat band does not have any degeneracy.

Finally, we comment on the anisotropic response to the external magnetic field. The magnetic mirror symmetry $\mathcal{T} M_{c a}$ is broken when we apply the magnetic field along the $b$ axis. Then, the zero-energy Majorana states disappear at the (100) edge, as expected [Fig. 14(b)]. This field angle dependence is attributed to the Ising character of the Majorana state. Similarly, the Majorana mode appears (disappears) at the (010) edge in the magnetic field along the $b$ axis ( $a$ axis), because the mirror symmetry along the $b c$ plane $\mathcal{M}_{b c}$ is preserved at the edge.

\section{SUMMARY AND DISCUSSION}

In this paper, we studied 2D multilayer SCs influenced by the layer-dependent RSOC. We showed that the odd-parity PDW state is stabilized by competing spin-orbit coupling and 
the orbital effect in the magnetic field along the $2 \mathrm{D}$ conducting plane. We also showed that the PDW state is a topological crystalline SC protected by the magnetic mirror symmetry when a small $p$-wave component is induced by the RSOC. The Majorana state has been demonstrated at the (100) edge [(010) edge] in the magnetic field along the $a$ axis ( $b$ axis).

Our finding paves the way toward realizing odd-parity superconductivity without a considerable pairing interaction in the spin-triplet channel. Although spin-triplet superconductivity is hardly stabilized in most SCs except for a few exceptions, our proposal provides an alternative way to create odd-parity $\mathrm{SC}$ by using the sublattice degree of freedom.

Indeed, recent developments in the technology of artificial heterostructures may enable the design of the odd-parity PDW state. Superconducting 2D electron systems have been fabricated in the oxide interfaces $\mathrm{SrTiO}_{3} / \mathrm{LaAlO}_{3}$ [62] and $\mathrm{SrTiO}_{3} / \mathrm{LaTiO}_{3}$ [63], gate-tuned $\mathrm{SrTiO}_{3}$ [64] and $\mathrm{MoS}_{2}$ [65], and the heavy-fermion superlattice $\mathrm{CeCoIn}_{5} / \mathrm{YbCoIn}_{5}$ [25]. It has been reported that interfacial (intrinsic) spin-orbit coupling significantly affects the superconducting state in $\mathrm{SrTiO}_{3}$ heterostructures [42,66-68] and $\mathrm{CeCoIn} / 5 / \mathrm{YbCoIn}_{5}$ $[28,29]\left(\mathrm{MoS}_{2}[69,70]\right)$. Furthermore, the multilayer structure has been artificially controlled in $\mathrm{CeCoIn}_{5} / \mathrm{YbCoIn}_{5}$ [29] and $\delta$-doped $\mathrm{SrTiO}_{3}$ [41]. Thus, we expect that odd-parity topological superconductivity will be created in these systems by tuning the multilayer structure and the magnetic field.

\section{ACKNOWLEDGMENTS}

The authors are grateful to Y. Iwasa, Y. Matsuda, T. Shibauchi, M. Sigrist, M. Shimozawa, and Y. Tada for fruitful discussions. This work was supported by the "Topological Quantum Phenomena" (No. 25103711) Grant-in Aid for Scientific Research on Innovative Areas from MEXT of Japan, and by JSPS KAKENHI Grants No. 24740230, No. 15K05164, No. 15H05745, and No. 15H05884 (J-Physics).
[1] M. Sigrist and K. Ueda, Phenomenological theory of unconventional superconductivity, Rev. Mod. Phys. 63, 239 (1991).

[2] D. J. Scalapino, The case for $d_{x^{2}-y^{2}}$ pairing in the cuprate superconductors, Phys. Rep. 250, 329 (1995).

[3] T. Moriya and K. Ueda, Spin fluctuations and high temperature superconductivity, Adv. Phys. 49, 555 (2000).

[4] Y. Yanase, T. Jujo, T. Nomura, H. Ikeda, T. Hotta, and $\mathrm{K}$. Yamada, Theory of superconductivity in strongly correlated electron systems, Phys. Rep. 387, 1 (2003).

[5] A. J. Leggett, A theoretical description of the new phases of liquid ${ }^{3} \mathrm{He}$, Rev. Mod. Phys. 47, 331 (1975).

[6] J. A. Sauls, The order parameter for the superconducting phases of $\mathrm{UPt}_{3}$, Adv. Phys. 43, 113 (1994).

[7] R. Joynt and L. Taillefer, The superconducting phases of $\mathrm{UPt}_{3}$, Rev. Mod. Phys. 74, 235 (2002).

[8] Y. Maeno, S. Kittaka, T. Nomura, S. Yonezawa, and K. Ishida, Evaluation of spin-triplet superconductivity in $\mathrm{Sr}_{2} \mathrm{RuO}_{4}$, J. Phys. Soc. Jpn. 81, 011009 (2012).

[9] Y. Yanase, S. Takamatsu, and M. Udagawa, Spin-orbit coupling and multiple phases in spin-triplet superconductor $\mathrm{Sr}_{2} \mathrm{RuO}_{4}$, J. Phys. Soc. Jpn. 83, 061019 (2014).

[10] N. Read and D. Green, Paired states of fermions in two dimensions with breaking of parity and time-reversal symmetries and the fractional quantum Hall effect, Phys. Rev. B 61, 10267 (2000).

[11] D. A. Ivanov, Non-Abelian Statistics of Half-Quantum Vortices in $p$-Wave Superconductors, Phys. Rev. Lett. 86, 268 (2001).

[12] A. Y. Kitaev, Unpaired Majorana fermions in quantum wires, Phys. Usp. 44, 131 (2001).

[13] A. P. Schnyder, S. Ryu, A. Furusaki, and A. W. W. Ludwig, Classification of topological insulators and superconductors in three spatial dimensions, Phys. Rev. B 78, 195125 (2008).

[14] A. Kitaev, in Periodic Table for Topological Insulators and Superconductors, AIP Conf. Proc. No. 1134 (AIP, New York, 2009), p. 22.

[15] M. Sato, Topological odd-parity superconductors, Phys. Rev. B 81, 220504 (2010).
[16] S. S. Saxena, P. Agarwal, K. Ahilan, F. M. Grosche, R. K. W. Haselwimmer, M. J. Steiner, E. Pugh, I. R. Walker, S. R. Julian, P. Monthoux, G. G. Lonzarich, A. Huxley, I. Sheikin, D. Braithwaite and J. Flouquet, Superconductivity on the border of itinerant-electron ferromagnetism in $\mathrm{UGe}_{2}$, Nature (London) 406, 587 (2000).

[17] D. Aoki and J. Flouquet, Ferromagnetism and superconductivity in uranium compounds, J. Phys. Soc. Jpn. 81, 011003 (2012).

[18] T. Hattori, Y. Ihara, Y. Nakai, K. Ishida, Y. Tada, S. Fujimoto, N. Kawakami, E. Osaki, K. Deguchi, N. K. Sato, and I. Satoh, Superconductivity Induced by Longitudinal Ferromagnetic Fluctuations in UCoGe, Phys. Rev. Lett. 108, 066403 (2012).

[19] M. H. Fischer, F. Loder, and M. Sigrist, Superconductivity and local noncentrosymmetry in crystal lattices, Phys. Rev. B 84, 184533 (2011).

[20] D. Maruyama, M. Sigrist, and Y. Yanase, Locally noncentrosymmetric superconductivity in multilayer systems, J. Phys. Soc. Jpn. 81, 034702 (2012).

[21] L. Fu and E. Berg, Odd-Parity Topological Superconductors: Theory and Application to $\mathrm{Cu}_{x} \mathrm{Bi}_{2} \mathrm{Se}_{3}$, Phys. Rev. Lett. 105, 097001 (2010).

[22] S. Nakosai, Y. Tanaka, and N. Nagaosa, Topological Superconductivity in Bilayer Rashba System, Phys. Rev. Lett. 108, 147003 (2012).

[23] T. Yoshida, M. Sigrist, and Y. Yanase, Pair-density wave states through spin-orbit coupling in multilayer superconductors, Phys. Rev. B 86, 134514 (2012).

[24] T. Yoshida, M. Sigrist, and Y. Yanase, Topological Crystalline Superconductivity in Locally Non-Centrosymmetric Multilayer Superconductors, Phys. Rev. Lett. 115, 027001 (2015).

[25] Y. Mizukami, H. Shishido, T. Shibauchi, M. Shimozawa, S. Yasumoto, D. Watanabe, M. Yamashita, H. Ikeda, T. Terashima, H. Kontani, and Y. Matsuda, Extremely strongcoupling superconductivity in artificial two-dimensional Kondo lattices, Nat. Phys. 7, 849 (2011). 
[26] K. Izawa, H. Yamaguchi, Y. Matsuda, H. Shishido, R. Settai, and Y. Onuki, Angular Position of Nodes in the Superconducting Gap of Quasi-2D. Heavy-Fermion Superconductor $\mathrm{CeCoIn}_{5}$, Phys. Rev. Lett. 87, 057002 (2001).

[27] T. Tayama, A. Harita, T. Sakakibara, Y. Haga, H. Shishido, R. Settai, and Y. Onuki, Unconventional heavy-fermion superconductor $\mathrm{CeCoIn}_{5}$ : Dc magnetization study at temperatures down to $50 \mathrm{mK}$, Phys. Rev. B 65, 180504 (2002).

[28] S. K. Goh, Y. Mizukami, H. Shishido, D. Watanabe, S. Yasumoto, M. Shimozawa, M. Yamashita, T. Terashima, Y. Yanase, T. Shibauchi, A. I. Buzdin, and Y. Matsuda, Anomalous Upper Critical Field in $\mathrm{CeCoIn}{ }_{5} / \mathrm{YbCoIn}_{5}$ Superlattices with a Rashba-Type Heavy Fermion Interface, Phys. Rev. Lett. 109, 157006 (2012).

[29] M. Shimozawa, S. K. Goh, R. Endo, R. Kobayashi, T. Watashige, Y. Mizukami, H. Ikeda, H. Shishido, Y. Yanase, T. Terashima, T. Shibauchi, and Y. Matsuda, Controllable Rashba Spin-Orbit Interaction in Artificially Engineered Superlattices Involving the Heavy-Fermion Superconductor CeCoIn ${ }_{5}$, Phys. Rev. Lett. 112, 156404 (2014).

[30] C. F. Miclea, M. Nicklas, D. Parker, K. Maki, J. L. Sarrao, J. D. Thompson, G. Sparn, and F. Steglich, Pressure Dependence of the Fulde-Ferrell-Larkin-Ovchinnikov State in $\mathrm{CeCoIn}_{5}$, Phys. Rev. Lett. 96, 117001 (2006).

[31] T. Yoshida, M. Sigrist, and Y. Yanase, Complex-stripe phases induced by staggered Rashba spin-orbit coupling, J. Phys. Soc. Jpn. 82, 074714 (2013).

[32] E. I. Rashba, Symmetry of bands in wurtzite-type crystals I. Symmetry of bands disregarding spin-orbit interaction, Sov. Phys. Solid State 1, 368 (1959); E. I. Rashba and V. I. Sheka, Symmetry of energy bands in crystals of wurtzite type II. Symmetry of bands with spin-orbit interaction included, Fiz. Tverd. Tela: Collected Papers 2, 162 (1959).

[33] C. Iniotakis, S. Fujimoto, and M. Sigrist, Fractional flux quanta at intrinsic metallic interfaces of noncentrosymmetric superconductors, J. Phys. Soc. Jpn. 77, 083701 (2008).

[34] E. Arahata, T. Neupert, and M. Sigrist, Spin currents and spontaneous magnetization at twin boundaries of noncentrosymmetric superconductors, Phys. Rev. B 87, 220504 (2013).

[35] M. Achermann, T. Neupert, E. Arahata, and M. Sigrist, Ginzburg-Landau description of twin boundaries in noncentrosymmetric superconductors, J. Phys. Soc. Jpn. 83, 044712 (2014).

[36] K. Aoyama, L. Savary, and M. Sigrist, Signatures of the helical phase in the critical fields at twin boundaries of noncentrosymmetric superconductors, Phys. Rev. B 89, 174518 (2014).

[37] P. Fulde and R. A. Ferrell, Superconductivity in a strong spinexchange field, Phys. Rev. 135, A550 (1964).

[38] A. I. Larkin and Y. N. Ovchinnikov, Nonuniform state of superconductors, Zh. Eksp. Teor. Fiz. 47, 1136 (1964) [Sov. Phys. JETP 20, 762 (1965)].

[39] Y. Yanase and M. Sigrist, Superconductivity and magnetism in non-centrosymmetric system: Application to $\mathrm{CePt}_{3} \mathrm{Si}$, J. Phys. Soc. Jpn. 77, 124711 (2008).

[40] Y. Matsuda and H. Shimahara, Fulde-Ferrell-LarkinOvchinnikov state in heavy fermion superconductors, J. Phys. Soc. Jpn. 76, 051005 (2007).

[41] H. Inoue, M. Kim, C. Bell, Y. Hikita, S. Raghu, and H. Y. Hwang, Tunable coupling of two-dimensional superconductors in bilayer $\mathrm{SrTiO}_{3}$ heterostructures, Phys. Rev. B 88, 241104 (2013).

[42] Y. Nakamura and Y. Yanase, Multi-orbital superconductivity in $\mathrm{SrTiO}_{3} / \mathrm{LaAlO}_{3}$ interface and $\mathrm{SrTiO}_{3}$ surface, J. Phys. Soc. Jpn. 82, 083705 (2013)

[43] X.-L. Qi and S.-C. Zhang, Topological insulators and superconductors, Rev. Mod. Phys. 83, 1057 (2011).

[44] Y. Tanaka, M. Sato, and N. Nagaosa, Symmetry and topology in superconductors-Odd-frequency pairing and edge states, J. Phys. Soc. Jpn. 81, 011013 (2012).

[45] F. Zhang, C. L. Kane, and E. J. Mele, Topological Mirror Superconductivity, Phys. Rev. Lett. 111, 056403 (2013).

[46] C.-K. Chiu, H. Yao, and S. Ryu, Classification of topological insulators and superconductors in the presence of reflection symmetry, Phys. Rev. B 88, 075142 (2013).

[47] T. Morimoto and A. Furusaki, Topological classification with additional symmetries from Clifford algebras, Phys. Rev. B 88, 125129 (2013).

[48] K. Shiozaki and M. Sato, Topology of crystalline insulators and superconductors, Phys. Rev. B 90, 165114 (2014).

[49] C.-K. Chiu and A. P. Schnyder, Classification of reflectionsymmetry-protected topological semimetals and nodal superconductors, Phys. Rev. B 90, 205136 (2014).

[50] Y. Ueno, A. Yamakage, Y. Tanaka, and M. Sato, SymmetryProtected Majorana Fermions in Topological Crystalline Superconductors: Theory and Application to $\mathrm{Sr}_{2} \mathrm{RuO}_{4}$, Phys. Rev. Lett. 111, 087002 (2013).

[51] T. Yoshida, M. Sigrist, and Y. Yanase, Parity-mixed superconductivity in locally non-centrosymmetric system, J. Phys. Soc. Jpn. 83, 013703 (2014).

[52] M. Sato and S. Fujimoto, Topological phases of noncentrosymmetric superconductors: Edge states, Majorana fermions, and non-Abelian statistics, Phys. Rev. B 79, 094504 (2009).

[53] M. Sato, Y. Tanaka, K. Yada, and T. Yokoyama, Topology of Andreev bound states with flat dispersion, Phys. Rev. B 83, 224511 (2011).

[54] K. Yada, M. Sato, Y. Tanaka, and T. Yokoyama, Surface density of states and topological edge states in noncentrosymmetric superconductors, Phys. Rev. B 83, 064505 (2011).

[55] A. P. Schnyder and S. Ryu, Topological phases and surface flat bands in superconductors without inversion symmetry, Phys. Rev. B 84, 060504(R) (2011).

[56] T. Mizushima, M. Sato, and K. Machida, Symmetry Protected Topological Order and Spin Susceptibility in Superfluid ${ }^{3} \mathrm{He}-\mathrm{B}$, Phys. Rev. Lett. 109, 165301 (2012).

[57] Y. Tsutsumi, M. Ishikawa, T. Kawakami, T. Mizushima, M. Sato, M. Ichioka, and K. Machida, $\mathrm{UPt}_{3}$ as a topological crystalline superconductor, J. Phys. Soc. Jpn. 82, 113707 (2013).

[58] S. Tewari and J. D. Sau, Topological Invariants for Spin-Orbit Coupled Superconductor Nanowires, Phys. Rev. Lett. 109, 150408 (2012).

[59] C. L. M. Wong and K. T. Law, Majorana Kramers doublets in $d_{x^{2}-y^{2}}$-wave superconductors with Rashba spin-orbit coupling, Phys. Rev. B 86, 184516 (2012).

[60] Y. Tanaka, T. Yokoyama, A. V. Balatsky, and N. Nagaosa, Theory of topological spin current in noncentrosymmetric superconductors, Phys. Rev. B 79, 060505 (2009).

[61] Y. Yanase and M. Sigrist, Non-centrosymmetric superconductivity and antiferromagnetic order: Microscopic discussion of $\mathrm{CePt}_{3} \mathrm{Si}$, J. Phys. Soc. Jpn. 76, 043712 (2007). 
[62] N. Reyren, S. Thiel, A. D. Caviglia, L. Fitting Kourkoutis, G. Hammerl, C. Richter, C. W. Schneider, T. Kopp, A.-S. Ruetschi, D. Jaccard, M. Gabay, D. A. Muller, J.-M. Triscone, and J. Mannhart, Superconducting interfaces between insulating oxides, Science 317, 1196 (2007).

[63] J. Biscaras, N. Bergeal, A. Kushwaha, T. Wolf, A. Rastogi, R. C. Budhani, and J. Lesueur, Two-dimensional superconductivity at a Mott insulator/band insulator interface $\mathrm{LaTiO}_{3} / \mathrm{SrTiO}_{3}$, Nat. Commun. 1, 89 (2010).

[64] K. Ueno, S. Nakamura, H. Shimotani, A. Ohtomo, N. Kimura, T. Nojima, H. Aoki, Y. Iwasa, and M. Kawasaki, Electric-fieldinduced superconductivity in an insulator, Nat. Mater. 7, 855 (2008).

[65] J. T. Ye, Y. J. Zhang, R. Akashi, M. S. Bahramy, R. Arita, and Y. Iwasa, Superconducting dome in a gate-tuned band insulator, Science 338, 1193 (2012).

[66] M. Ben Shalom, M. Sachs, D. Rakhmilevitch, A. Palevski, and Y. Dagan, Tuning Spin-Orbit Coupling and Superconductivity at the $\mathrm{SrTiO}_{3} / \mathrm{LaAlO}_{3}$ Interface: A Magnetotransport Study, Phys. Rev. Lett. 104, 126802 (2010).

[67] A. D. Caviglia, M. Gabay, S. Gariglio, N. Reyren, C. Cancellieri, and J. M. Triscone, Tunable Rashba Spin-Orbit Interaction at Oxide Interfaces, Phys. Rev. Lett. 104, 126803 (2010).

[68] K. Michaeli, A. C. Potter, and P. A. Lee, Superconducting and Ferromagnetic Phases in $\mathrm{SrTiO}_{3} / \mathrm{LaAlO}_{3}$ Oxide Interface Structures: Possibility of Finite Momentum Pairing, Phys. Rev. Lett. 108, 117003 (2012).

[69] Y. Saito, Y. Nakamura, M. S. Bahramy, Y. Kohama, Y. Kasahara, Y. Nakagawa, M. Onga, M. Tokunaga, T. Nojima, Y. Yanase, and Y. Iwasa, Superconductivity protected by spin-valley locking in gate-tuned $\mathrm{MoS}_{2}$, arXiv:1506.04146.

[70] J. M. Lu, O. Zeliuk, I. Leermakers, N. F. Q. Yuan, U. Zeitler, K. T. Law, and J. T. Ye, Two dimensional Ising superconductivity in gated $\mathrm{MoS}_{2}$, arXiv:1506.07620. 